\title{
An ILP improvement procedure for the Open Vehicle Routing Problem
}

\author{
Majid Salari, Paolo Toth *, Andrea Tramontani \\ DEIS, University of Bologna, Viale Risorgimento 2, 40136 Bologna, Italy
}

\section{A R T I C L E I N F O}

Keywords:

Integer Linear Programming

Local search

Heuristics

Open Vehicle Routing Problem

\begin{abstract}
A B S T R A C T
We address the Open Vehicle Routing Problem (OVRP), a variant of the "classical" (capacitated and distance constrained) Vehicle Routing Problem (VRP) in which the vehicles are not required to return to the depot after completing their service. We present a heuristic improvement procedure for OVRP based on Integer Linear Programming (ILP) techniques. Given an initial feasible solution to be possibly improved, the method follows a destruct-and-repair paradigm, where the given solution is randomly destroyed (i.e., customers are removed in a random way) and repaired by solving an ILP model, in the attempt of finding a new improved feasible solution. The overall procedure can be considered as a general framework which could be extended to cover other variants of Vehicle Routing Problems. We report computational results on benchmark instances from the literature. In several cases, the proposed algorithm is able to find the new best known solution for the considered instances.
\end{abstract}

(c) 2010 Elsevier Ltd. All rights reserved.

\section{Introduction}

We address the Open Vehicle Routing Problem (OVRP), a variant of the "classical" (capacitated and distance constrained) Vehicle Routing Problem (VRP) in which the vehicles are not required to return to the depot after completing their service. OVRP can be formally stated as follows. We are given a central depot and a set of $n$ customers, which are associated with the nodes of a complete undirected graph $G=(V, E)$ (where $V=\{0,1, \ldots, n\}$, node 0 represents the depot and $V \backslash\{0\}$ is the set of customers). Each edge $e \in E$ has an associated finite cost $c_{e} \geq 0$ and each customer $v \in V \backslash\{0\}$ has a demand $q_{v}>0$ (with $q_{0}=0$ ). A fleet of $m$ identical vehicles is located at the depot, each one with a fixed cost $F$, a capacity $Q$ and a total distance-traveled (duration) limit $D$. The customers must be served by at most $m$ Hamiltonian paths (open routes), each path associated with one vehicle, starting at the depot and ending at one of the customers. Each route must have a duration (computed as the sum of the edge costs in the route) not exceeding the given limit $D$ of the vehicles, and can visit a subset $S$ of customers whose total demand $\sum_{v \in S} q_{v}$ does not exceed the given capacity $Q$. The problem consists of finding a feasible solution covering (i.e., visiting) exactly once all the customers and having a minimum overall cost, computed as the sum of the traveled edge costs plus the fixed costs associated with the vehicles used to serve the customers. OVRP is known to be

\footnotetext{
* Corresponding author. Tel.: +39051 2093028; fax: +390512093073.

E-mail addresses: majid.salari2@unibo.it (M. Salari), paolo.toth@unibo.it

(P. Toth), andrea.tramontani@unibo.it (A. Tramontani).
}

$\mathcal{N P}$-hard in the strong sense, as it generalizes the Bin Packing Problem and the Hamiltonian Path Problem.

In this paper we present a heuristic improvement procedure for OVRP based on Integer Linear Programming (ILP) techniques. Given an initial feasible solution to be possibly improved, the procedure iteratively performs the following steps: (a) randomly select several customers from the current solution, and build the restricted solution obtained from the current one by extracting (i.e., short-cutting) the selected customers; (b) reallocate the extracted customers to the restricted solution by solving an ILP problem, in the attempt of finding a new improved feasible solution. This method has been proposed by De Franceschi et al. [7] and deeply investigated by Toth and Tramontani [27] in the context of the classical VRP. Here, we consider a simpler version of this approach, which exploits no particular feature of the addressed problem. The method follows a destruct-and-repair paradigm, where the current solution is randomly destroyed (i.e., customers are removed in a random way) and repaired by following ILP techniques. Hence, the overall procedure can be considered as a general framework which could be extended to cover other variants of Vehicle Routing Problems.

The notion of using ILP techniques to improve a feasible solution of a combinatorial optimization problem has emerged in several papers in the last few years. Addressing the split delivery VRP, Archetti et al. [2] developed a heuristic algorithm that integrates tabu search with ILP by solving integer programs to explore promising parts of the solution space identified by a tabu search heuristic. A similar approach has been presented by Archetti et al. [1] for an inventory routing problem. Hewitt et al. [15] proposed to solve the capacitated fixed charge network flow problem by combining exact and heuristic approaches. In this 
case as well a key ingredient of the method is to use ILP to improve feasible solutions found during the search. Finally, the idea of exploiting ILP to explore promising neighborhoods of feasible solutions has been also investigated in the context of general purpose integer programs; see, e.g., Fischetti and Lodi [10] and Danna et al. [6]. The methods presented in [6,10] are currently embedded in the commercial mixed integer programming solver Cplex [16].

The paper is organized as follows. Section 2 recalls the main works proposed in the literature for OVRP. In Section 3 we describe a neighborhood for OVRP and the ILP model which allows to implicitly define and explore the presented neighborhood. The implementation of the heuristic improvement procedure is given in Section 4, while Section 5 reports the computational experiments on benchmark capacitated OVRP instances from the literature (with/without distance constraints), comparing the presented method with the most effective metaheuristic techniques proposed for OVRP. Some conclusions are finally drawn in Section 6.

\section{Literature review}

The classical VRP is a fundamental combinatorial optimization problem which has been widely studied in the literature (see, e.g., Toth and Vigo [28] and Cordeau et al. [5]). At first glance, having open routes instead of closed ones looks like a minor change, and in fact OVRP can be also formulated as a VRP on a directed graph, by fixing to 0 the cost of each arc entering the depot. However, if the undirected case is considered, the open version turns out to be more general than the closed one. Indeed, as shown by Letchford et al. [17], any closed VRP on $n$ customers in a complete undirected graph can be transformed into an OVRP on $n$ customers, but there is no transformation in the reverse direction. Further, there are many practical applications in which OVRP naturally arises. This happens, of course, when a company does not own a vehicle fleet, and hence customers are served by hired vehicles which are not required to come back to the depot (see, e.g., Tarantilis et al. [26]). But the open model also arises in pick-up and delivery applications, where each vehicle starts at the depot, delivers to a set of customers and then it is required to visit the same customers in reverse order, picking up items that have to be backhauled to the depot. An application of this type is described in Schrage [23]. Further areas of application, involving the planning of train services and of school bus routes, are reported by Fu et al. [13].

OVRP has recently received an increasing attention in the literature. Exact branch-and-cut and branch-cut-and-price approaches have been proposed, respectively, by Letchford et al. [17] and Pessoa et al. [19], addressing the capacitated problem with no distance constraints and no empty routes allowed (i.e., $D=\infty$ and exactly $m$ vehicles must be used). Heuristic and metaheuristic algorithms usually take into account both capacity and distance constraints, and consider the number of routes as a decision variable. In particular, an unlimited number of vehicles is supposed to be available (i.e., $m=\infty$ ) and the objective function is generally to minimize the number of used vehicles first and the traveling cost second, assuming that the fixed cost of an additional vehicle always exceeds any traveling cost that could be saved by its use (i.e., considering $F=\infty$ ). However, several authors address as well the variant in which there are no fixed costs associated with the vehicles (i.e., $F=0$ ) and hence the objective function is to minimize the total traveling cost with no attention to the number of used vehicles (see, e.g., Tarantilis et al. [26]). Considering capacity constraints only (i.e., taking $D=\infty$ ), Sariklis and Powell [22] propose a two-phase heuristic which first assigns customers to clusters and then builds a Hamiltonian path for each cluster, Tarantilis et al. [24] describe a population-based heuristic, while Tarantilis et al. [25,26] present threshold accepting metaheuristics. Taking into account both capacity and distance constraints, Brandão [3], Fu et al. [13,14] and Derigs and Reuter [8] propose tabu search heuristics, Li et al. [18] describe a record-to-record travel heuristic, Pisinger and Ropke [20] present an adaptive large neighborhood search heuristic which follows a destruct-and-repair paradigm, while Fleszar et al. [12] propose a variable neighborhood search heuristic.

\section{Reallocation model}

Let $z$ be a feasible solution of the OVRP defined on $G$. For any given node subset $\mathcal{F} \subset V \backslash\{0\}$, we define $z(\mathcal{F})$ as the restricted solution obtained from $z$ by extracting (i.e., by short-cutting) all the nodes $v \in \mathcal{F}$. Let $\mathcal{R}$ be the set of routes in the restricted solution, $\mathcal{I}=\mathcal{I}(z, \mathcal{F})$ the set of all the edges in $z(\mathcal{F})$, and $\mathcal{S}=\mathcal{S}(\mathcal{F})$ the set of all the sequences which can be obtained through the recombination of nodes in $\mathcal{F}$ (i.e., the set of all the elementary paths in $\mathcal{F}$ ). Each edge $i \in \mathcal{I}$ is viewed as a potential insertion point which can allocate one or more nodes in $\mathcal{F}$ through at most one sequence $s \in \mathcal{S}$. We say that the insertion point $i=(a, b) \in \mathcal{I}$ allocates the nodes $\left\{v_{j} \in \mathcal{F}\right.$ : $j=1, \ldots, h\}$ through the sequence $s=\left(v_{1}, v_{2}, \ldots, v_{h}\right) \in \mathcal{S}$, if the edge $(a, b)$ in the restricted solution is replaced by the edges $\left(a, v_{1}\right),\left(v_{1}, v_{2}\right), \ldots,\left(v_{h}, b\right)$ in the new feasible solution. Since the restricted routes, as well as the final ones, are open paths starting at the depot, in addition to the edges of the restricted solution we also consider the insertion points (called appending insertion points in the following) $i=\left(p_{r}, 0\right)$, where $p_{r}$ denotes the last customer visited by route $r \in \mathcal{R}$, which allow to append any sequence to the last customer of any restricted route. Further, empty routes in the restricted solution are associated with insertion points $(0,0)$.

For each sequence $s \in \mathcal{S}, c(s)$ and $q(s)$ denote, respectively, the cost of the elementary path corresponding to $s$ and the sum of the demands of the nodes in $s$. For each insertion point $i=(a, b) \in \mathcal{I}$ and for each sequence $s=\left(v_{1}, v_{2}, \ldots, v_{h}\right) \in \mathcal{S}, \gamma_{s i}$ denotes the extra-cost (i.e., the extra-distance) for assigning sequence $s$ to insertion point $i$ in its best possible orientation (i.e., $\gamma_{s i}:=c(s)-c_{a b}+\min \left\{c_{a v_{1}}+c_{v_{h} b}, c_{a v_{h}}+\right.$ $\left.\left.c_{v_{1} b}\right\}\right)$. Note that, for the appending insertion points $i=\left(p_{r}, 0\right), \gamma_{s i}$ is computed as $c(s)+\min \left\{c_{p_{r} v_{1}}, c_{p_{r} v_{h}}\right\}$. The extra-cost for assigning the sequence $s$ to the insertion point $i=(0,0)$ associated with an empty route is simply $c(s)+\min \left\{c_{0 v_{1}}, c_{0 v_{h}}\right\}$. For each route $r \in \mathcal{R}, \mathcal{I}(r)$ denotes the set of insertion points associated with $r$, while $\tilde{q}(r)$ and $\tilde{c}(r)$ denote, respectively, the total demand and the total distance computed for route $r$, still in the restricted solution.

For each $i \in \mathcal{I}, \mathcal{S}_{i} \subseteq \mathcal{S}$ denotes a sequence subset containing the sequences which can be allocated to the specific insertion point $i$. The definition of $\mathcal{S}_{i}$ will be discussed later in this section. Then, a neighborhood of the given solution $z$ can be formulated (and explored) by solving an ILP problem (denoted as the Reallocation Model) based on the decision variables

$x_{s i}= \begin{cases}1 & \text { if sequence } s \in \mathcal{S}_{i} \text { is allocated to insertion point } i \in \mathcal{I} \\ 0 & \text { otherwise, }\end{cases}$

which reads as follows:

$\sum_{r \in \mathcal{R}} \tilde{c}(r)+\min \sum_{i \in \mathcal{I} s \in \mathcal{S}_{i}} \gamma_{s i} x_{s i}$

subject to

$\sum_{i \in \mathcal{I} s \in \mathcal{S}_{i}(v)} x_{s i}=1, \quad v \in \mathcal{F}$,

$\sum_{s \in \mathcal{S}_{i}} x_{s i} \leq 1, \quad i \in \mathcal{I}$ 
$\sum_{i \in \mathcal{I}(r)} \sum_{s \in \mathcal{S}_{i}} q(s) x_{s i} \leq Q-\tilde{q}(r), \quad r \in \mathcal{R}$,

$\sum_{i \in \mathcal{I}(r)} \sum_{s \in \mathcal{S}_{i}} \gamma_{s i} x_{s i} \leq D-\tilde{c}(r), \quad r \in \mathcal{R}$,

$x_{s i} \in\{0,1\}, \quad i \in \mathcal{I}, s \in \mathcal{S}_{i}$,

where, for any $i \in \mathcal{I}$ and $v \in \mathcal{F}, \mathcal{S}_{i}(v) \subseteq \mathcal{S}_{i}$ denotes the set of sequences covering customer $v$ which can be allocated to insertion point $i$. The objective function (2), to be minimized, gives the traveling cost of the final OVRP solution. Constraints (3) impose that each extracted node belongs to exactly one of the selected sequences, i.e., that it is covered exactly once in the final solution. Constraints (4) avoid to allocate two or more sequences to the same insertion point. Finally, constraints (5) and (6) impose that each route in the final solution fulfills the capacity and distance restrictions, respectively. Note that, if there is a non-null fixed cost $F$ associated with the vehicles, it can be taken into account by simply adding $F$ to the cost of the edges incident at the depot node.

The Reallocation Model (2)-(7) defines a neighborhood of a given solution $z$ which depends on the extracted nodes $\mathcal{F}$ and on the subsets $\mathcal{S}_{i}(i \in \mathcal{I})$. In particular, for any given $\mathcal{F}$, the choice of $\mathcal{S}_{i}$ is a key factor in order to allow an effective exploration of the solution space in the neighborhood of the given solution. The subsets $\mathcal{S}_{i}$ are built by following a column generation approach: we initialize the Linear Programming (LP) relaxation of the Reallocation Model (LP-RM) with a subsets of variables with small insertion cost, and afterwards we iteratively solve the column generation problem associated with LP-RM, adding other variables with small reduced cost. The overall procedure for building the subsets $\mathcal{S}_{i}$ can be described as follows.

1. (Initialization) For each insertion point $i=\left(a_{i}, b_{i}\right) \in \mathcal{I}$, initialize subset $\mathcal{S}_{i}$ with the basic sequence extracted from $i$ (i.e., the, possibly empty, sequence of nodes connecting node $a_{i}$ and $b_{i}$ in the given solution $z$ ) plus the feasible singleton sequence with the minimum insertion cost (i.e., the sequence $(v)$, with $v \in \mathcal{F}$, with the minimum extra-cost among all the singleton sequences which can be allocated to $i$ without violating the capacity and distance restrictions for the restricted route containing $i$ ). Initialize LP-RM with the initial set of variables corresponding to the current subsets $\mathcal{S}_{i}$, and solve LP-RM.

2. (Column generation) For each insertion point $i \in \mathcal{I}$, solve the column generation problem associated with $i$, adding to $\mathcal{S}_{i}$ all the sequences $s$ corresponding to elementary paths in $\mathcal{F}$, whose associated variables $x_{s i}$ have a reduced cost $r c_{s i}$ under a given threshold $R C_{\max }$ (i.e., variables $x_{s i}$ such that $r c_{s i} \leq R C_{\max }$ ). If at least one sequence/variable has been added, solve the new LP-RM and repeat step 2 . Otherwise terminate.

For any fixed insertion point $i \in \mathcal{I}$, the column generation problem associated with $i$ in LP-RM is a Resource Constrained Elementary Shortest Path Problem (RCESPP), which usually arises in the Set Partitioning formulation of the classical VRP (see, e.g., Feillet et al. [9] and Righini and Salani [21]). Here, for each insertion point $i \in \mathcal{I}$, we solve the corresponding RCESPP through a simple greedy heuristic, with the aim of finding as many variables with small reduced cost as possible. Hashing techniques are used to avoid the generation of duplicated variables.

Note that each subset $\mathcal{S}_{i}$ contains the basic sequence extracted from insertion point $i$, and hence the current solution can always be obtained as a new feasible solution of the Reallocation Model.

\subsection{Column generation for the Reallocation Model}

Let $\pi_{v}^{1}, \pi_{i}^{2}, \pi_{r}^{3}$ and $\pi_{r}^{4}$ be the dual variables associated, respectively, with constraints (3)-(6) in LP-RM, where $v \in \mathcal{F}, i \in \mathcal{I}$ and $r \in \mathcal{R}$, and denote with $\tilde{\pi}=\left(\tilde{\pi}_{v}^{1}, \tilde{\pi}_{i}^{2}, \tilde{\pi}_{r}^{3}, \tilde{\pi}_{r}^{4}\right)$ the optimal dual solution of LP-RM. For any fixed $i=\left(a_{i}, b_{i}\right) \in \mathcal{I}$, consider the directed graph $\tilde{G}(i, \tilde{\pi})=$ $\left(V_{i}, A_{i}\right)$, with $V_{i}:=\left\{a_{i}, b_{i}\right\} \cup \mathcal{F}$ and $A_{i}:=\left\{(v, w): v \in V_{i}, w \in V_{i}\right\} \backslash\left\{\left(a_{i}, b_{i}\right)\right.$, $\left.\left(b_{i}, a_{i}\right)\right\}$.Associate with each $\operatorname{arc} a=(v, w) \in A_{i}, w \neq 0$, a weight $\theta_{a}$ equal to the cost of the corresponding edge $e=(v, w)$ in the graph $G$, while set $\theta_{a}:=0$ for each arc $a=(v, 0) \in A_{i}$, if $0 \in V_{i}$. Associate with each arc $a \in A_{i}$ a cost $c_{a}^{\prime}=\theta_{a}\left(1-\tilde{\pi}_{r_{i}}^{4}\right)$, and associate with each node $v \in \mathcal{F}$ a weight $q_{v}$ and a cost $q_{v}^{\prime}=-\left(\tilde{\pi}_{v}^{1}+q_{v} \tilde{\pi}_{r_{i}}^{3}\right)$. Then, let $P=\left(V_{P}, A_{P}\right)$ be an elementary path $\left(a_{i}, v_{1}, \ldots, v_{h}, b_{i}\right)$ connecting nodes $a_{i}$ and $b_{i}$ in $\tilde{G}(i, \tilde{\pi})$, where $V_{P}:=\left\{v_{1}, \ldots, v_{h}\right\} \subseteq V_{i}$ and $A_{P}:=\left\{\left(a_{i}, v_{1}\right), \ldots,\left(v_{h}, b_{i}\right)\right\} \subseteq A_{i}$. We say that $P$ is a feasible path if

$\sum_{v \in V_{P}} q_{v} \leq Q-\tilde{d}\left(r_{i}\right), \quad \sum_{a \in A_{P}} \theta_{a} \leq D-\tilde{c}\left(r_{i}\right)+c_{i}$,

where $c_{i}$ denotes the cost of insertion point $i=\left(a_{i}, b_{i}\right)$, while the cost of the path is

$c^{\prime}(P)=\sum_{a \in A_{P}} c_{a}^{\prime}+\sum_{v \in V_{P}} q_{v}^{\prime}$.

Any sequence $s=\left(v_{1}, \ldots, v_{h}\right) \in \mathcal{S}$ is clearly associated with the elementary path $\left(a_{i}, v_{1}, \ldots, v_{h}, b_{i}\right)$ in $\tilde{G}(i, \tilde{\pi})$. The reduced cost $r c_{s i}$ of variable $x_{s i}$ in LP-RM is defined by

$r c_{s i}:=\gamma_{s i}-\sum_{v \in V_{P}} \tilde{\pi}_{v}^{1}-\tilde{\pi}_{i}^{2}-q(s) \tilde{\pi}_{r_{i}}^{3}-\gamma_{s i} \tilde{\pi}_{r_{i}}^{4}$

and can easily be rewritten as

$r c_{s i}:=-\tilde{\pi}_{i}^{2}-c_{i}\left(1-\tilde{\pi}_{r_{i}}^{4}\right)+\sum_{a \in A_{P}} c_{a}^{\prime}+\sum_{v \in V_{P}} q_{v}^{\prime}$.

Hence, the following proposition holds:

Proposition 1. For any $i=\left(a_{i}, b_{i}\right) \in \mathcal{I}$, the column generation problem associated with $i$ in LP-RM is the problem of finding an elementary feasible path $P$ from $a_{i}$ to $b_{i}$ in $\tilde{G}(i, \tilde{\pi})$, with cost $c^{\prime}(P)<\tilde{\pi}_{i}^{2}+c_{i}\left(1-\tilde{\pi}_{r_{i}}^{4}\right)$.

As described above, the column generation problem for LP-RM associated with any insertion point $i \in \mathcal{I}$ is a Resource Constrained Elementary Shortest Path Problem (RCESPP) defined on graph $\tilde{G}(i, \tilde{\pi})$, whose size strictly depends on $|\mathcal{F}|$. The orientation of $\tilde{G}(i, \tilde{\pi})$ is required only when the considered $i=\left(a_{i}, b_{i}\right) \in \mathcal{I}$ is an appending insertion point (i.e., $b_{i}$ is the depot node). Even in this case, the column generation problem could be addressed on a mixed graph, where only the edges incident at the depot are replaced by directed arcs (of different cost and weight) entering and leaving the depot. In the general case, $\tilde{G}(i, \tilde{\pi})$ contains negative cycles (i.e., cycles in which the sum of the costs $c_{a}^{\prime}$ associated with the arcs and the costs $q_{v}^{\prime}$ associated with the nodes is negative): indeed, while dual variables $\pi_{i}^{2}, \pi_{r}^{3}, \pi_{r}^{4}$ are non-positive, dual variables $\pi_{v}^{1}$ are free and usually assume positive values. Positive values of variables $\pi_{v}^{1}$ can lead to negative node costs $q_{v}^{\prime}$ and to negative cycles in graph $\tilde{G}(i, \tilde{\pi})$. Therefore, the column generation problem in LP-RM is strongly $\mathcal{N} \mathcal{P}$-hard.

In order to find a promising set of variables for the Reallocation Model in a short computing time, we solve the RCESPP associated with each insertion point through a simple heuristic. We say that a node $v \in \mathcal{F}$ is feasible for $i \in \mathcal{I}$ if the singleton sequence $(v)$ can be allocated to $i$ without violating the capacity and distance restrictions on the restricted route $r_{i}$. For any given insertion point $i=\left(a_{i}, b_{i}\right) \in \mathcal{I}$, we first build a reduced graph $\tilde{G}(i, \tilde{\pi})$, obtained by considering only nodes $a_{i}, b_{i}$ and the $n f$ feasible nodes of $\mathcal{F}$ with smallest insertion cost (i.e., the $n f$ feasible nodes $v_{k} \in \mathcal{F}, k=1, \ldots, n f$, whose associated singleton sequences $\left(v_{k}\right)$ have the smallest 
extra-cost for $i$ ). At each iteration of the column generation step described in Section 3, $n f$ is uniformly randomly generated in $\left[n f_{\min }, n f_{\text {max }}\right]$. Then, on the reduced graph $\tilde{G}(i, \tilde{\pi})$, we apply the following simple heuristic:

1. Find an initial feasible path $P=\left(a_{i}, v, b_{i}\right)$, in $\tilde{G}(i, \tilde{\pi})$.

2. Evaluate all the 1-1 feasible exchanges between each node $w \in V_{i} \backslash V_{P}$ and each node $v \in V_{P}$, and select the best one (with respect to the cost of the corresponding path); if this exchange leads to an improvement, perform it and repeat step 2.

3. Evaluate all the feasible insertions of each node $w \in V_{i} \backslash V_{P}$ in each arc $\left(v_{1}, v_{2}\right) \in A_{P}$ and select the best one; if no feasible insertion exists, terminate; otherwise, force such an insertion even if it leads to a worse path and repeat step 2 .

Whenever a new path in $\tilde{G}(i, \tilde{\pi})$ is generated, the corresponding sequence is added to $\mathcal{S}_{i}$ if the reduced cost of $x_{s i}$ is smaller than a given threshold $R C_{\max }$.

\section{Heuristic improvement procedure}

The Reallocation Model described in the previous section allows for exploring a neighborhood of a given feasible solution, depending on the choice of the extracted customers in $\mathcal{F}$. We propose a heuristic improvement procedure for OVRP, based on model (2)-(7), which iteratively explores different neighborhoods of the current solution. Given an initial feasible solution $z_{0}$ for OVRP (taken from the literature or found by any heuristic method), the procedure works as follows.

1. (Initialization) Set $k t:=0$ and $k p:=0$. Take $z_{0}$ as the incumbent solution and initialize the current solution $z_{c}$ as $z_{c}:=z_{0}$.

2. (Node selection) Build set $\mathcal{F}$ by selecting each customer with a probability $p$.

3. (Node extraction) Extract the nodes selected in the previous step from the current solution $z_{c}$ and construct the corresponding restricted OVRP solution $z_{c}(\mathcal{F})$, obtained by short-cutting the extracted nodes.

4. (Reallocation) Define the subsets $\mathcal{S}_{i}\left(i \in \mathcal{I}\left(z_{c}, \mathcal{F}\right)\right)$ as described in Section 3. Build the corresponding Reallocation Model (2)-(7) and solve the model by using a general-purpose ILP solver. Once an optimal ILP solution has been found, construct the corresponding new OVRP solution and possibly update $z_{c}$ and $z_{0}$.

5. (Termination) Set $k t:=k t+1$. If $k t=K T_{\max }$, terminate.

6. (Perturbation) If $z_{c}$ has been improved in the last iteration, set $k p:=0$; otherwise set $k p:=k p+1$. If $k p=K P_{\max }$, "perturb" the current solution $z_{c}$ and set $k p:=0$. In any case, repeat step 2 .

The procedure performs $K T_{\max }$ iterations and at each iteration explores a randomly generated neighborhood of the current solution $z_{c}$. However, if $z_{c}$ is not improved for $K P_{\max }$ consecutive iterations, we introduce a random perturbation (see Step 6) in order to move to a different area of the solution space, so as to enforce the diversification of the search. In particular, when performing a Perturbation Step, we randomly extract $n p$ customers from $z_{c}$ (with $n p$ uniformly randomly chosen in [ $\left.n p_{\min }, n p_{\max }\right]$ and with each customer having the same probability to be extracted), and reinsert each extracted customer, in turn, in its best feasible position. If a customer cannot be inserted in any currently non-empty route (due to the capacity and/or distance restrictions), a new route is created to allocate the customer. In general, when performing the Perturbation Step, several customers cannot be inserted in the non-empty routes of the current solution, and hence the new perturbed solution can use more vehicles than the current one.

\section{Computational results}

The performance of the Heuristic Improvement Procedure (HIP) described in the previous sections was evaluated on the 16 benchmark instances usually addressed in the literature, taken from Christofides et al. [4] (instances $\mathrm{C}_{1}-\mathrm{C} 14$ ) and from Fisher [11] (instances $\mathrm{F} 11-\mathrm{F} 12$ ), and on the 8 large scale benchmark instances proposed by Li et al. [18], and also addressed by Derigs and Reuter [8] (instances 01-08). The number of customers of $\mathrm{C} 1-\mathrm{C} 14$ and $\mathrm{F} 11-\mathrm{F} 12$ ranges from 50 to $199 . \mathrm{C} 1-\mathrm{C} 5, \mathrm{C} 11-\mathrm{C} 12$ and F11-F12 have only capacity constraints, while $\mathrm{C} 6-\mathrm{C} 10$ and $\mathrm{C} 13-\mathrm{C} 14$ are the same instances as $\mathrm{C} 1-\mathrm{C} 5$ and $\mathrm{C} 11-\mathrm{C} 12$, respectively, but with both capacity and distance constraints. Instances $01-08$ have no distance restrictions and a number of customers varying from 200 to 480 . As usual, for the problems with distance constraints, the route duration limit $D$ is taken as the original value for the classical VRP multiplied by 0.9 .

HIP needs an initial solution to be given, which in principle could be computed through any available constructive heuristic algorithm. We decided to run HIP starting from an extremely good feasible solution available from the literature (in several cases, the best known solution reported in the literature), with the aim of attempting to improve it (this is of course impossible if the initial solution is provably optimal, as it is the case for some of them). In particular, we considered as initial solutions the ones obtained by Fu et al. [13,14], Pisinger and Ropke [20], Derigs and Reuter [8] and Fleszar et al. [12].

HIP has been tested on a Pentium IV 3.4 GHz with 1 GByte RAM, running under Microsoft Windows XP Operative System, and has been coded in $\mathrm{C}++$ with Microsoft Visual $\mathrm{C}+6.0$ compiler. The ILP solver used in the experiments is ILOG Cplex 10.0 [16]. HIP setting depends on the parameters $R C_{\max }, p, n f_{\min }, n f_{\max }, n p_{\min }, n p_{\max }$, and on the number of iterations $K P_{\max }$ and $K T_{\max }$. Although these parameters could be tuned considering the edge costs and the particular characteristics of each tested instance, we preferred to run all the experiments with a fixed set of parameters: $R C_{\max }=1$, $p=0.5$ (i.e., $50 \%$ of the customers are selected on average), $n f_{\min }=15, \quad n f_{\max }=25, \quad n p_{\min }=15, \quad n p_{\max }=25, \quad K P_{\max }=50$ and $K T_{\max }=5000$ (i.e., we perform globally 5000 iterations, and the current solution is perturbed if it cannot be improved for 50 consecutive iterations). Further, since several authors address the problem considering as objective function the minimization of the number of vehicles first and of the traveling cost second (i.e., assuming $F=\infty$ ), while other authors considered as objective function the minimization of the traveling cost (i.e., $F=0$ ), we decided to run HIP without allowing to change the number of vehicles used in the initial solution. However, as stated in Section 4 , the Perturbation Step often requires additional routes to be created (to preserve the feasibility of the solution). In such cases, we add a small penalty $\theta$ to the cost of the edges incident at the depot, in order to force HIP to "recover" the solution in the following iterations. After some preliminary tests, we decided to fix $\theta=12$ for the considered instances. Finally, HIP is a randomized algorithm and hence the computational results may depend on the randomization. For each tested instance (and each initial solution), we considered five runs of the algorithm corresponding to five different seeds for generating the random numbers.

The computational results are reported in Tables $1-3$. All the CPU times are expressed in seconds, and all the solution costs have been computed in double precision.

Table 1 reports the computational results on the 16 instances $\mathrm{C} 1-\mathrm{C} 14$ and F11-F12 obtained by starting from the solutions 
Table 1

Computational results on the "classical" 16 benchmark instances starting from the solutions by Fu et al. [13,14].

\begin{tabular}{|c|c|c|c|c|c|c|c|c|c|c|c|c|c|c|c|c|c|c|c|c|}
\hline \multirow[t]{2}{*}{$\mathrm{Pb}$} & \multirow[t]{2}{*}{$m$} & \multirow[t]{2}{*}{ P.best } & \multicolumn{2}{|l|}{ Initial } & \multicolumn{2}{|l|}{ Run 1} & \multicolumn{2}{|l|}{ Run 2} & \multicolumn{2}{|l|}{ Run 3} & \multicolumn{2}{|l|}{ Run 4} & \multicolumn{2}{|l|}{ Run 5} & \multicolumn{2}{|l|}{ Best } & \multicolumn{2}{|l|}{ Worst } & \multicolumn{2}{|l|}{ Average } \\
\hline & & & Cost & $\% \mathrm{dev}$ & Cost & $\% \operatorname{dev}$ & Cost & $\% \operatorname{dev}$ & Cost & \%dev & Cost & \%dev & Cost & $\% \operatorname{dev}$ & Cost & \%dev & Cost & $\% \operatorname{dev}$ & Cost & $\% \operatorname{dev}$ \\
\hline C2 & 10 & 567.14 & 567.14 & 0.00 & - & 0.00 & - & 0.00 & - & 0.00 & - & 0.00 & - & 0.00 & 567.14 & 0.00 & 567.14 & 0.00 & 567.14 & 0.00 \\
\hline C3 & 8 & ${ }^{*} 639.74$ & $\overline{641.88}$ & 0.33 & *639.74 & 0.00 & 640.42 & 0.11 & 640.42 & 0.11 & 640.42 & 0.11 & 640.42 & 0.11 & ${ }^{*} 639.74$ & 0.00 & $\overline{640.42}$ & 0.11 & 640.28 & 0.08 \\
\hline C4 & 12 & 733.13 & 738.94 & 0.79 & 733.13 & 0.00 & 733.13 & 0.00 & 733.13 & 0.00 & 733.13 & 0.00 & 733.13 & 0.00 & 733.13 & 0.00 & 733.13 & 0.00 & 733.13 & 0.00 \\
\hline C5 & 17 & 869.25 & 878.95 & 1.12 & $\overline{868.81}$ & -0.05 & $\overline{868.81}$ & -0.05 & $\overline{868.81}$ & -0.05 & $\overline{868.81}$ & -0.05 & $\overline{868.81}$ & -0.05 & $\overline{868.81}$ & -0.05 & $\overline{868.81}$ & -0.05 & $\overline{868.81}$ & -0.05 \\
\hline C6 & 6 & 412.96 & 412.96 & 0.00 & - & 0.00 & - & 0.00 & - & 0.00 & - & 0.00 & - & 0.00 & 412.96 & 0.00 & 412.96 & 0.00 & 412.96 & 0.00 \\
\hline C7 & 11 & 568.49 & $\overline{568.49}$ & 0.00 & - & 0.00 & - & 0.00 & - & 0.00 & - & 0.00 & - & 0.00 & $\overline{568.49}$ & 0.00 & $\overline{568.49}$ & 0.00 & $\overline{568.49}$ & 0.00 \\
\hline C8 & 9 & 644.63 & 646.31 & 0.26 & 644.63 & 0.00 & 644.63 & 0.00 & 644.63 & 0.00 & 644.63 & 0.00 & 644.63 & 0.00 & 644.63 & 0.00 & 644.63 & 0.00 & 644.63 & 0.00 \\
\hline C9 & 14 & 756.14 & 761.28 & 0.68 & $\overline{756.14}$ & 0.00 & $\overline{756.14}$ & 0.00 & $\overline{756.38}$ & 0.03 & $\overline{756.38}$ & 0.03 & $\overline{756.14}$ & 0.00 & $\overline{756.14}$ & 0.00 & $\overline{756.38}$ & 0.03 & $\overline{756.24}$ & 0.01 \\
\hline C10 & 17 & 875.07 & 903.10 & 3.20 & $\overline{878.54}$ & 0.40 & $\overline{879.13}$ & 0.46 & 877.47 & 0.27 & 880.25 & 0.59 & 879.68 & 0.53 & $\overline{877.47}$ & 0.27 & 880.25 & 0.59 & 879.01 & 0.45 \\
\hline $\mathrm{C} 11$ & 7 & 682.12 & 717.15 & 5.14 & 683.64 & 0.22 & 685.20 & 0.45 & 685.20 & 0.45 & 682.83 & 0.10 & 682.83 & 0.10 & 682.83 & 0.10 & 685.20 & 0.45 & 683.94 & 0.27 \\
\hline $\mathrm{C} 12$ & 10 & ${ }^{*} 534.24$ & 534.71 & 0.09 & *534.24 & 0.00 & *534.24 & 0.00 & *534.24 & 0.00 & *534.24 & 0.00 & *534.24 & 0.00 & *534.24 & 0.00 & *534.24 & 0.00 & *534.24 & 0.00 \\
\hline $\mathrm{C} 13$ & 12 & 896.50 & 917.90 & 2.39 & $\overline{894.19}$ & -0.26 & 897.37 & 0.10 & 896.66 & 0.02 & 897.37 & 0.10 & $8 \overline{96.14}$ & -0.04 & 894.19 & -0.26 & 897.37 & 0.10 & $8 \overline{96.35}$ & -0.02 \\
\hline C14 & 11 & 591.87 & 600.66 & 1.49 & 591.87 & 0.00 & 591.87 & 0.00 & 591.87 & 0.00 & 591.87 & 0.00 & 591.87 & 0.00 & 591.87 & 0.00 & 591.87 & 0.00 & 591.87 & 0.00 \\
\hline F12 & 7 & 769.66 & 777.07 & 0.96 & $\overline{769.55}$ & -0.01 & $\overline{770.38}$ & 0.09 & $\overline{769.55}$ & -0.01 & $\overline{770.38}$ & 0.09 & $\overline{770.38}$ & 0.09 & $\overline{769.55}$ & -0.01 & $\overline{770.38}$ & 0.09 & $\overline{770.05}$ & 0.05 \\
\hline Avg. & & & & 1.18 & & 0.02 & & 0.08 & & 0.06 & & 0.07 & & 0.05 & & 0.00 & & 0.09 & & 0.06 \\
\hline $\mathrm{Pb}$ & & & t.time & & t.time & b.time & t.time & b.time & t.time & b.time & t.time & b.time & t.time & b.time & & & & & t.time & b.time \\
\hline $\mathrm{C} 2$ & & & 7.8 & & 84.2 & 84.2 & 86.5 & 86.5 & 80.2 & 80.2 & 81.9 & 81.9 & 89.6 & 89.6 & & & & & 84.5 & 84.5 \\
\hline C3 & & & 23.2 & & 119.9 & 106.0 & 110.9 & 74.7 & 117.9 & 56.8 & 139.9 & 132.0 & 118.8 & 88.3 & & & & & 121.5 & 91.6 \\
\hline C4 & & & 6.8 & & 156.6 & 21.2 & 157.8 & 0.6 & 154.7 & 0.4 & 198.1 & 0.8 & 164.1 & 0.7 & & & & & 166.3 & 4.7 \\
\hline C5 & & & 61.9 & & 220.3 & 10.3 & 220.0 & 11.6 & 228.5 & 32.7 & 277.8 & 12.9 & 225.5 & 12.5 & & & & & 234.4 & 16.0 \\
\hline C6 & & & 0.6 & & 45.1 & 45.1 & 43.2 & 43.2 & 49.0 & 49.0 & 44.7 & 44.7 & 42.2 & 42.2 & & & & & 44.8 & 44.8 \\
\hline C7 & & & 6.0 & & 83.1 & 83.1 & 87.7 & 87.7 & 83.2 & 83.2 & 79.8 & 79.8 & 79.5 & 79.5 & & & & & 82.7 & 82.7 \\
\hline C8 & & & & & 136.2 & 0.1 & 135.9 & 0.1 & 144.9 & 3.2 & 177.7 & 2.6 & 144.7 & 0.1 & & & & & 147.9 & 1.2 \\
\hline C9 & & & 46.6 & & 255.5 & 102.7 & 265.5 & 7.9 & 247.7 & 195.7 & 312.1 & 299.6 & 259.2 & 18.3 & & & & & 268.0 & 124.8 \\
\hline C10 & & & 51.9 & & 460.2 & 323.9 & 477.7 & 35.9 & 513.0 & 453.1 & 505.9 & 474.5 & 497.8 & 366.5 & & & & & 490.9 & 330.8 \\
\hline C11 & & & 23.1 & & 198.8 & 165.8 & 199.8 & 40.4 & 229.8 & 225.7 & 329.6 & 204.2 & 201.4 & 200.0 & & & & & 231.9 & 167.2 \\
\hline C12 & & & 4.2 & & 94.0 & 1.6 & 98.2 & 73.2 & 99.1 & 89.5 & 106.2 & 16.6 & 107.8 & 92.1 & & & & & 101.1 & 54.6 \\
\hline $\mathrm{C} 13$ & & & 82.1 & & 1165.3 & 475.0 & 1004.9 & 201.4 & 1180.5 & 685.0 & 1146.5 & 725.2 & 1396.5 & 1230.9 & & & & & 1178.7 & 663.5 \\
\hline C14 & & & 2.5 & & 354.7 & 293.8 & 339.2 & 88.6 & 321.3 & 33.7 & 366.6 & 337.3 & 453.9 & 435.8 & & & & & 367.1 & 237.8 \\
\hline F12 & & & 28.4 & & 148.2 & 77.8 & 158.0 & 74.9 & 154.8 & 70.7 & 169.3 & 30.9 & 168.2 & 67.5 & & & & & 159.7 & 64.4 \\
\hline Avg. & & & 24.7 & & 251.6 & 127.9 & 241.8 & 59.1 & 257.5 & 147.1 & 281.2 & 174.5 & 282.1 & 194.6 & & & & & 262.8 & 140.6 \\
\hline
\end{tabular}

CPU times are expressed in seconds. 
Table 2

Computational results on the "classical" 16 benchmark instances starting from the best available solutions.

\begin{tabular}{|c|c|c|c|c|c|c|c|c|c|c|c|c|c|c|c|c|c|c|c|c|}
\hline \multirow[t]{2}{*}{$\mathrm{Pb}$} & \multirow[t]{2}{*}{$m$} & \multirow[t]{2}{*}{ P.best } & \multicolumn{2}{|l|}{ Initial } & \multicolumn{2}{|l|}{ Run 1} & \multicolumn{2}{|l|}{ Run 2} & \multicolumn{2}{|l|}{ Run 3} & \multicolumn{2}{|l|}{ Run 4} & \multicolumn{2}{|l|}{ Run 5} & \multicolumn{2}{|l|}{ Best } & \multicolumn{2}{|l|}{ Worst } & \multicolumn{2}{|l|}{ Average } \\
\hline & & & Cost & $\%$ dev & Cost & $\%$ dev & Cost & \%dev & Cost & $\%$ dev & Cost & $\% \operatorname{dev}$ & Cost & \%dev & Cost & $\%$ dev & Cost & $\%$ dev & Cost & $\% \operatorname{dev}$ \\
\hline $\mathrm{C} 2$ & 10 & 567.14 & 567.14 & 0.00 & - & 0.00 & - & 0.00 & - & 0.00 & - & 0.00 & - & 0.00 & 567.14 & 0.00 & 567.14 & 0.00 & 567.14 & 0.00 \\
\hline C4 & 12 & 733.13 & $\overline{733.13}$ & 0.00 & - & 0.00 & - & 0.00 & - & 0.00 & - & 0.00 & - & 0.00 & $\overline{733.13}$ & 0.00 & $\overline{733.13}$ & 0.00 & $\overline{733.13}$ & 0.00 \\
\hline C5 & 16 & 879.37 & $\overline{896.08}$ & 1.90 & 892.37 & 1.48 & 892.37 & 1.48 & 892.37 & 1.48 & 892.37 & 1.48 & 892.37 & 1.48 & $\overline{892.37}$ & 1.48 & $\overline{892.37}$ & 1.48 & $\overline{892.37}$ & 1.48 \\
\hline C5 & 17 & 869.24 & $\underline{869.24}$ & 0.00 & 868.93 & -0.04 & 868.93 & -0.04 & 869.00 & -0.03 & 868.93 & -0.04 & 868.93 & -0.04 & 868.93 & -0.04 & 869.00 & -0.03 & 868.94 & -0.03 \\
\hline C6 & 6 & 412.96 & $\overline{412.96}$ & 0.00 & - & 0.00 & - & 0.00 & - & 0.00 & - & 0.00 & - & 0.00 & 412.96 & 0.00 & 412.96 & 0.00 & 412.96 & 0.00 \\
\hline C7 & 10 & 583.19 & $\overline{583.19}$ & 0.00 & - & 0.00 & - & 0.00 & - & 0.00 & - & 0.00 & - & 0.00 & $\overline{583.19}$ & 0.00 & $\overline{583.19}$ & 0.00 & $\overline{583.19}$ & 0.00 \\
\hline C7 & 11 & 568.49 & 568.49 & 0.00 & - & 0.00 & - & 0.00 & - & 0.00 & - & 0.00 & - & 0.00 & $\overline{568.49}$ & 0.00 & $\overline{568.49}$ & 0.00 & $\overline{568.49}$ & 0.00 \\
\hline C8 & 9 & 644.63 & 644.63 & 0.00 & - & 0.00 & - & 0.00 & - & 0.00 & - & 0.00 & - & 0.00 & 644.63 & 0.00 & 644.63 & 0.00 & 644.63 & 0.00 \\
\hline C9 & 13 & 757.84 & $\overline{757.84}$ & 0.00 & 757.73 & -0.01 & 757.69 & -0.02 & 757.70 & -0.02 & 757.73 & -0.01 & 757.73 & -0.01 & 757.69 & -0.02 & $\overline{757.73}$ & -0.01 & $\overline{757.72}$ & -0.02 \\
\hline C9 & 14 & 756.14 & $\overline{756.14}$ & 0.00 & - & 0.00 & - & 0.00 & - & 0.00 & - & 0.00 & - & 0.00 & 756.14 & 0.00 & 756.14 & 0.00 & 756.14 & 0.00 \\
\hline $\mathrm{C} 10$ & 17 & 875.07 & 875.07 & 0.00 & 874.71 & -0.04 & 874.71 & -0.04 & 874.71 & -0.04 & 874.71 & -0.04 & 874.71 & -0.04 & $\overline{874.71}$ & -0.04 & $\overline{874.71}$ & -0.04 & $\overline{874.71}$ & -0.04 \\
\hline C11 & 7 & 682.12 & $\overline{682.12}$ & 0.00 & - & 0.00 & - & 0.00 & - & 0.00 & - & 0.00 & - & 0.00 & 682.12 & 0.00 & 682.12 & 0.00 & 682.12 & 0.00 \\
\hline C13 & 11 & 904.04 & $\overline{904.04}$ & 0.00 & 899.16 & -0.54 & 899.16 & -0.54 & 899.16 & -0.54 & 899.16 & -0.54 & 899.16 & -0.54 & $\overline{899.16}$ & -0.54 & $\overline{899.16}$ & -0.54 & $\overline{899.16}$ & -0.54 \\
\hline C13 & 12 & 896.50 & $\overline{917.90}$ & 2.39 & 894.19 & -0.26 & 897.37 & 0.10 & 896.66 & 0.02 & 897.37 & 0.10 & 896.14 & -0.04 & 894.19 & -0.26 & 897.37 & 0.10 & 896.35 & -0.02 \\
\hline C14 & 11 & 591.87 & 591.87 & 0.00 & - & 0.00 & - & 0.00 & - & 0.00 & - & 0.00 & - & 0.00 & 591.87 & 0.00 & 591.87 & 0.00 & 591.87 & 0.00 \\
\hline C14 & 12 & 581.81 & 581.81 & 0.00 & - & 0.00 & - & 0.00 & - & 0.00 & - & 0.00 & - & 0.00 & $\underline{581.81}$ & 0.00 & $\underline{581.81}$ & 0.00 & 581.81 & 0.00 \\
\hline F12 & 7 & 769.66 & $\overline{769.66}$ & 0.00 & 769.55 & -0.01 & 769.55 & -0.01 & - & 0.00 & 769.55 & -0.01 & - & 0.00 & $\overline{769.55}$ & -0.01 & $\overline{769.66}$ & 0.00 & $\overline{769.59}$ & -0.01 \\
\hline Avg. & & & & 0.25 & & 0.03 & & 0.05 & & 0.05 & & 0.06 & & 0.05 & & 0.03 & & 0.06 & & 0.05 \\
\hline $\mathrm{Pb}$ & & & Source & & t.time & b.time & t.time & b.time & t.time & b.time & t.time & b.time & t.time & b.time & & & & & t.time & b.time \\
\hline $\mathrm{C} 2$ & & & {$[12,14,20]$} & & 84.2 & 84.2 & 86.5 & 86.5 & 80.2 & 80.2 & 81.9 & 81.9 & 89.6 & 89.6 & & & & & 84.5 & 84.5 \\
\hline C4 & & & {$[12,20]$} & & 151.2 & 151.2 & 137.5 & 137.5 & 123.2 & 123.2 & 164.5 & 164.5 & 153.5 & 153.5 & & & & & 146.0 & 146.0 \\
\hline C5 & & & [20] & & 450.2 & 276.5 & 480.4 & 237.5 & 463.4 & 237.5 & 434.0 & 237.5 & 518.3 & 237.5 & & & & & 469.3 & 245.3 \\
\hline C5 & & & [8] & & 275.2 & 130.2 & 247.5 & 195.4 & 278.1 & 21.4 & 260.5 & 205.0 & 255.8 & 166.9 & & & & & 263.4 & 143.8 \\
\hline C6 & & & {$[12,14,20]$} & & 45.1 & 45.1 & 43.2 & 43.2 & 49.0 & 49.0 & 44.7 & 44.7 & 42.2 & 42.2 & & & & & 44.8 & 44.8 \\
\hline C7 & & & [20] & & 80.6 & 80.6 & 86.1 & 86.1 & 73.1 & 73.1 & 81.2 & 81.2 & 85.2 & 85.2 & & & & & 81.2 & 81.2 \\
\hline C7 & & & [14] & & 83.1 & 83.1 & 87.7 & 87.7 & 83.2 & 83.2 & 79.8 & 79.8 & 79.5 & 79.5 & & & & & 82.7 & 82.7 \\
\hline C8 & & & [12] & & 136.8 & 136.8 & 142.2 & 142.2 & 137.2 & 137.2 & 130.0 & 130.0 & 143.9 & 143.9 & & & & & 138.0 & 138.0 \\
\hline C9 & & & [20] & & 412.5 & 33.9 & 404.6 & 330.6 & 372.9 & 0.2 & 355.4 & 11.0 & 413.0 & 6.9 & & & & & 391.7 & 76.5 \\
\hline C9 & & & [8] & & 243.4 & 243.4 & 213.9 & 213.9 & 221.9 & 221.9 & 227.6 & 227.6 & 267.1 & 267.1 & & & & & 234.8 & 234.8 \\
\hline $\mathrm{C} 10$ & & & [8] & & 454.7 & 2.6 & 390.1 & 2.4 & 344.0 & 1.7 & 395.6 & 4.2 & 387.6 & 0.7 & & & & & 394.4 & 2.3 \\
\hline C11 & & & {$[12,20]$} & & 178.3 & 178.3 & 183.4 & 183.4 & 181.0 & 181.0 & 183.4 & 183.4 & 165.3 & 165.3 & & & & & 178.3 & 178.3 \\
\hline C13 & & & [12] & & 959.3 & 6.3 & 1022.0 & 133.3 & 1030.3 & 6.6 & 1027.2 & 4.6 & 980.5 & 78.6 & & & & & 1003.9 & 45.9 \\
\hline C13 & & & [14] & & 1165.3 & 475.0 & 1004.9 & 201.4 & 1180.5 & 685.0 & 1146.5 & 725.2 & 1396.5 & 1230.9 & & & & & 1178.7 & 663.5 \\
\hline C14 & & & {$[12,20]$} & & 276.3 & 276.3 & 293.8 & 293.8 & 263.6 & 263.6 & 294.4 & 294.4 & 301.1 & 301.1 & & & & & 285.8 & 285.8 \\
\hline C14 & & & [8] & & 364.2 & 364.2 & 304.0 & 304.0 & 310.5 & 310.5 & 290.4 & 290.4 & 354.1 & 354.1 & & & & & 324.6 & 324.6 \\
\hline F12 & & & [12] & & 142.2 & 56.6 & 157.2 & 103.4 & 143.9 & 143.9 & 137.2 & 64.2 & 129.7 & 129.7 & & & & & 142.0 & 99.6 \\
\hline Avg. & & & & & 323.7 & 154.4 & 310.9 & 163.7 & 313.9 & 154.1 & 313.8 & 166.4 & 339.0 & 207.8 & & & & & 320.2 & 169.3 \\
\hline
\end{tabular}

CPU times are expressed in seconds. 
Table 3

Computational results on the eight large scale benchmark instances starting from the solutions by Derigs and Reuter [8]

\begin{tabular}{|c|c|c|c|c|c|c|c|c|c|c|c|c|c|c|c|c|c|c|c|c|}
\hline \multirow[t]{2}{*}{$\mathrm{Pb}$} & \multirow[t]{2}{*}{$m$} & \multirow[t]{2}{*}{ P.best } & \multicolumn{2}{|l|}{ Initial } & \multicolumn{2}{|l|}{ Run 1} & \multicolumn{2}{|l|}{ Run 2} & \multicolumn{2}{|l|}{ Run 3} & \multicolumn{2}{|l|}{ Run 4} & \multicolumn{2}{|l|}{ Run 5} & \multicolumn{2}{|l|}{ Best } & \multicolumn{2}{|l|}{ Worst } & \multicolumn{2}{|l|}{ Average } \\
\hline & & & Cost & $\% \operatorname{dev}$ & Cost & $\%$ dev & Cost & $\%$ dev & Cost & $\%$ dev & Cost & $\%$ dev & Cost & $\% \operatorname{dev}$ & Cost & $\%$ dev & Cost & $\%$ dev & Cost & $\%$ dev \\
\hline 01 & 5 & 6018.52 & 6018.52 & 0.00 & - & 0.00 & - & 0.00 & - & 0.00 & - & 0.00 & - & 0.00 & 6018.52 & 0.00 & 6018.52 & 0.00 & 6018.52 & 0.00 \\
\hline $\mathrm{O} 2$ & 9 & 4584.55 & $\overline{4584.69}$ & 0.00 & 4573.53 & -0.24 & 4573.53 & -0.24 & 4573.53 & -0.24 & 4573.53 & -0.24 & 4573.53 & -0.24 & $\overline{4573.53}$ & -0.24 & $\overline{4573.53}$ & -0.24 & $\overline{4573.53}$ & -0.24 \\
\hline 03 & 7 & 7731.46 & 7731.46 & 0.00 & - & 0.00 & - & 0.00 & - & 0.00 & - & 0.00 & - & 0.00 & 7731.46 & 0.00 & 7731.46 & 0.00 & 7731.46 & 0.00 \\
\hline 04 & 10 & 7260.59 & $\overline{7260.59}$ & 0.00 & 7259.81 & -0.01 & 7253.91 & -0.09 & 7253.91 & -0.09 & 7253.20 & -0.10 & 7251.74 & -0.12 & $\overline{7251.74}$ & -0.12 & $\overline{7259.81}$ & -0.01 & $\overline{7254.51}$ & -0.08 \\
\hline 05 & 9 & 9167.19 & $\overline{9167.19}$ & 0.00 & 9165.40 & -0.02 & 9156.74 & -0.11 & 9157.42 & -0.11 & 9159.22 & -0.09 & 9159.22 & -0.09 & 9156.74 & -0.11 & 9165.40 & -0.02 & 9159.6 & -0.08 \\
\hline O6 & 9 & 9803.80 & $\overline{9805.45}$ & 0.02 & - & 0.02 & - & 0.02 & - & 0.02 & - & 0.02 & 9804.25 & 0.00 & 9804.25 & 0.00 & 9805.45 & 0.02 & 9805.21 & 0.01 \\
\hline 07 & 10 & 10348.57 & 10348.57 & 0.00 & 10344.37 & -0.04 & 10344.37 & -0.04 & 10344.37 & -0.04 & 10344.37 & -0.04 & 10344.37 & -0.04 & 10344.37 & -0.04 & 10344.37 & -0.04 & 10344.37 & -0.04 \\
\hline 08 & 10 & 12420.16 & $\overline{12420.16}$ & 0.00 & - & 0.00 & - & 0.00 & - & 0.00 & - & 0.00 & - & 0.00 & $\underline{12420.16}$ & 0.00 & $\underline{12420.16}$ & 0.00 & $\underline{12420.16}$ & 0.00 \\
\hline Avg. & & & & 0.00 & & -0.04 & & -0.06 & & -0.06 & & -0.06 & & -0.06 & & -0.06 & & -0.04 & & -0.05 \\
\hline $\mathrm{Pb}$ & & & t.time & & t.time & b.time & t.time & b.time & t.time & b.time & t.time & b.time & t.time & b.time & & & & & t.time & b.time \\
\hline 01 & & & 467.0 & & 182.2 & 182.2 & 191.5 & 191.5 & 174.0 & 174.0 & 168.5 & 168.5 & 175.9 & 175.9 & & & & & 178.4 & 178.4 \\
\hline $\mathrm{O} 2$ & & & 467.0 & & 284.0 & 34.6 & 298.6 & 233.0 & 302.8 & 89.2 & 313.0 & 63.3 & 395.5 & 360.6 & & & & & 318.8 & 156.1 \\
\hline 03 & & & 4047.0 & & 304.6 & 304.6 & 279.6 & 279.6 & 300.6 & 300.6 & 295.5 & 295.5 & 296.8 & 296.8 & & & & & 295.4 & 295.4 \\
\hline $\mathrm{O} 4$ & & & 927.0 & & 438.9 & 34.4 & 405.5 & 387.5 & 437.6 & 72.6 & 421.7 & 219.9 & 406.3 & 385.4 & & & & & 422.0 & 220.0 \\
\hline 05 & & & 1186.0 & & 499.6 & 41.4 & 479.1 & 270.8 & 513.9 & 496.5 & 550.3 & 173.6 & 479.5 & 210.5 & & & & & 504.5 & 238.6 \\
\hline O6 & & & 1231.0 & & 581.3 & 581.3 & 590.4 & 590.4 & 637.4 & 637.4 & 620.1 & 620.1 & 590.8 & 361.1 & & & & & 604.0 & 558.1 \\
\hline 07 & & & 3190.0 & & 653.0 & 8.6 & 631.8 & 23.8 & 661.6 & 420.9 & 743.5 & 306.6 & 619.7 & 387.2 & & & & & 661.9 & 229.4 \\
\hline 08 & & & 1969.0 & & 623.6 & 623.6 & 635.2 & 635.2 & 668.9 & 668.9 & 653.7 & 653.7 & 647.9 & 647.9 & & & & & 645.9 & 645.9 \\
\hline Avg. & & & 1685.5 & & 445.9 & 226.3 & 438.9 & 326.5 & 462.1 & 357.5 & 470.8 & 312.7 & 451.6 & 353.2 & & & & & 453.9 & 315.2 \\
\hline
\end{tabular}

CPU times are expressed in seconds. 
provided by $\mathrm{Fu}$, Eglese and $\mathrm{Li}$ and obtained through the algorithm proposed in [13]. In some cases, several solutions are provided for the same instance, obtained by using slightly different versions of their algorithm, with the same number of routes and different traveling cost. Among the different solutions for the same instance, we considered as initial solution for HIP the best one provided. For instances $\mathrm{C} 1$ and $\mathrm{F} 11$, all the solutions available from [13,14] are provably optimal (see, e.g., Letchford et al. [17]) and cannot be further improved. Thus, these instances were not considered in this set of experiments. The upper part of the table reports the solutions found by HIP. The first column gives the instance name $(\mathrm{Pb})$. Columns 2 and 3 report the number of vehicles used in the initial solution $(m)$ and the cost of the best known solution using the same number of vehicles (P.best). Columns 4 and 5 report the cost of the initial solution (cost) and the corresponding percentage deviation w.r.t. the best known value (\%dev), computed as $100 *$ (cost-P.best)/P.best. Then, for each of the 5 runs of the algorithm, we report the final solution cost provided by HIP and the corresponding percentage deviation (again computed w.r.t. the best known value). When HIP was not able to improve on the initial solution, we mark with a "-_" the final solution cost. Finally, we report the best, the worst and the average result out of the five different runs. Final solution costs equal to the previously best known ones are underlined, new best solutions are in bold face, while provably optimal solutions, taken from Letchford et al. [17], are marked with an *. The lower part of the table gives the computing times. First, we report the overall $\mathrm{CPU}$ time of the algorithm corresponding to the initial solution, obtained on a Pentium IV $3 \mathrm{GHz}$. These times have been taken from [14]. However, the cost of the initial solution for instance $c 8$ is better than the ones reported in [14], and hence for this initial solution we did not report the corresponding computing time. Then, for each run of the algorithm, we report the overall computing time required to perform all the 5000 iterations (t.time) and the CPU time required to reach the final solution (b.time). For a "fair" calculation of the average values, when HIP was not able to improve on the initial solution we considered b.time equal to the overall computing time. Finally, the last two columns give the average CPU times (i.e., average t.time and average b.time) out of the five different runs.

Table 2 reports the computational results on the same instances by starting from the best available solutions among the ones obtained by Fu et al. [13,14], Pisinger and Ropke [20], Derigs and Reuter [8] and Fleszar et al. [12]. The table has the same structure as Table 1, but column 2 in the lower part of the table reports the source of the initial solution used in the experiments. For instances $\mathrm{C} 5, \mathrm{C} 7, \mathrm{C} 9, \mathrm{C} 13$ and $\mathrm{C} 14$, the best available solutions for the case $F=\infty$ and the case $F=0$ are different. In such cases, we considered both the solutions as initial solutions for HIP. For instances $\mathrm{C} 1, \mathrm{C} 3, \mathrm{C} 12$ and $\mathrm{F} 11$, all the solutions available from $[8,12,20]$ are provably optimal and hence these instances were not considered in this set of experiments.

Finally, Table 3 reports the computational results on the 8 large scale instances $01-08$ by starting from the solutions provided by Derigs and Reuter [8]. The table has the same structure as Table 1 , but the CPU time related to the initial solution (column 2 in the lower part of the table) was obtained on a Pentium IV $2.8 \mathrm{GHz}$.

The tables show that HIP is able to improve even extremely good quality solutions, obtained by some of the most effective metaheuristic techniques proposed for OVRP. It is worth noting that the solutions and the CPU times provided by Fu et al. [13,14] and reported in Table 1 are the best ones from among 20 runs of the corresponding randomized algorithm with different seeds. Hence, taking into account the different performance of the processors used for testing the different algorithms, the overall computing time required by HIP is comparable with the others reported in the tables, and in several cases the final improved solution is found very quickly. Our test-bed concerns in practice 35 different, non-provably optimal, initial solutions which could be possibly improved, corresponding to 22 different instances. By considering the best result from among the five different runs executed for each of these 35 initial solutions, HIP improves on the initial solution in 22 cases. For these cases, HIP reaches 6 times the previously best known solution (provably optimal in two cases), while finds 12 times a new best solution. Considering the 13 initial solutions which HIP does not improve, it is worth noting that all these solutions are the best known ones in the literature (for the case $F=\infty$ or $F=0$ ). Looking at the different runs executed for each initial solution, we can note that in some cases the results depend on the seed used for the random generator. However, the method is overall quite consistent since, by considering all the tested initial solutions, the average computing time and the average final percentage deviation are only slightly affected by the choice of the seed.

In order to look for possible better solutions, we performed some additional experiments. In particular, after the first 5000 iterations, we ran HIP for 2000 more iterations with a slightly different parameter setting. Starting from the solutions provided by Fu et al. [14], for instance C5 with 17 vehicles, after 5220 iterations and $237.4 \mathrm{~s}$ HIP found a solution of cost 868.44 that corresponds to a further improvement on the previous best known solution. Finally, still starting from the solutions by Fu et al. [14], we ran HIP with a different tuning of parameter $p$, to investigate how the neighborhood size affects the overall performance of the method, both in terms of quality of the solutions found and of CPU time. Let $z_{\text {avg }}(\bar{p})$ be the average final solution cost obtained on the 14 instances $\mathrm{C} 2-\mathrm{C} 14$ and $\mathrm{F} 12$ with $p=\bar{p}$, and let time $\operatorname{avg}_{\mathrm{p}}(\bar{p})$ be the corresponding average CPU time in seconds. With $p=0.3,0.5$ and 0.7 we obtained the following results: $z_{\text {avg }}(0.3)=684.55$ and ttime $_{\text {avg }}(0.3)=71.9, \quad z_{\text {avg }}(0.5)=681.94$ and ttime $e_{\text {avg }}(0.5)=262.8$, $z_{\text {avg }}(0.7)=683.32$ and ttime $e_{\text {avg }}(0.7)=460.0$. As expected, the average CPU time consistently increases with the number of extracted customers, while the best solution costs are obtained with the default setting of $p$ (i.e., $p=0.5$ ), thus indicating that extracting too many customers leads in general to worse solutions (i.e., $\left.z_{\text {avg }}(0.7)>z_{\text {avg }}(0.5)\right)$. This is not completely surprising, and it is essentially due to the column generation heuristic, which falls in troubles in finding good variables for the Reallocation Model when the current solution has been almost completely "destroyed" by the removal of too many customers.

As previously seen, the proposed algorithm is able to improve on high-quality initial solutions. However, a natural question concerns the effectiveness of the method if the initial solution is instead a "bad-quality" solution. To answer this question, we implemented a modified version of the tabu search algorithm proposed by $\mathrm{Fu}$ et al. [13] (we refer the reader to [13] for a detailed description of this algorithm). More precisely, we first computed an initial random (and typically infeasible) solution, and then we applied only 200 iterations of the tabu search algorithm, with the aim of quickly finding a feasible solution, possibly "far" from the good ones. The computational results provided by HIP on the 16 instances $\mathrm{C} 1-\mathrm{C} 14$ and $\mathrm{F} 11-\mathrm{F} 12$ when starting from such initial solutions are reported in Table 4.

The table has the same structure as Table 1 and shows that HIP is quite effective even when the initial solution is not a goodquality solution. First, we can note that all the solutions are improved by all the five different runs. Further, even in this case the method is quite consistent, as all the five different runs provide on average very similar results, both in terms of quality of the solutions found and of CPU time. Finally, considering all the instances and all the different runs, the average behavior of the 
Table 4

Computational results on the "classical" 16 benchmark instances starting from "bad initial solutions".

\begin{tabular}{|c|c|c|c|c|c|c|c|c|c|c|c|c|c|c|c|c|c|c|c|c|}
\hline \multirow[t]{2}{*}{$\mathrm{Pb}$} & \multirow[t]{2}{*}{$m$} & \multirow[t]{2}{*}{ P.best } & \multicolumn{2}{|l|}{ Initial } & \multicolumn{2}{|l|}{ Run 1} & \multicolumn{2}{|l|}{ Run 2} & \multicolumn{2}{|l|}{ Run 3} & \multicolumn{2}{|l|}{ Run 4} & \multicolumn{2}{|l|}{ Run 5} & \multicolumn{2}{|l|}{ Best } & \multicolumn{2}{|l|}{ Worst } & \multicolumn{2}{|l|}{ Average } \\
\hline & & & Cost & $\% \operatorname{dev}$ & Cost & \%dev & Cost & $\%$ dev & Cost & \%dev & Cost & \%dev & Cost & \%dev & Cost & \%dev & Cost & $\%$ dev & Cost & $\% \operatorname{dev}$ \\
\hline $\mathrm{C} 1$ & 5 & *416.06 & 467.80 & 12.44 & 417.37 & 0.31 & 417.36 & 0.31 & $* 416.06$ & 0.00 & $* 416.06$ & 0.00 & 417.37 & 0.31 & $* 416.06$ & 0.00 & 417.37 & 0.31 & 416.84 & 0.19 \\
\hline C2 & 11 & 564.06 & 657.07 & 16.49 & 564.06 & 0.00 & 564.06 & 0.00 & $5 \overline{64.06}$ & 0.00 & $5 \overline{64.06}$ & 0.00 & 564.06 & 0.00 & $5 \overline{64.06}$ & 0.00 & 564.06 & 0.00 & 564.06 & 0.00 \\
\hline C3 & 8 & *639.74 & 768.93 & 20.19 & $\overline{642.14}$ & 0.38 & $\overline{642.14}$ & 0.38 & $\overline{642.98}$ & 0.51 & $\overline{642.14}$ & 0.38 & $\overline{643.75}$ & 0.63 & $\overline{642.14}$ & 0.38 & $\overline{643.75}$ & 0.63 & $\overline{642.63}$ & 0.45 \\
\hline C4 & 12 & 733.13 & 1069.38 & 45.86 & 738.05 & 0.67 & 741.75 & 1.18 & 748.63 & 2.11 & 742.11 & 1.22 & 744.15 & 1.50 & 738.05 & 0.67 & 748.63 & 2.11 & 742.94 & 1.34 \\
\hline C5 & 17 & 869.24 & 1449.20 & 66.72 & 887.40 & 2.09 & 879.89 & 1.23 & 882.12 & 1.48 & 887.48 & 2.10 & 887.85 & 2.14 & 879.89 & 1.23 & 887.85 & 2.14 & 884.95 & 1.81 \\
\hline C6 & 6 & 412.96 & 444.98 & 7.75 & 416.84 & 0.94 & 412.96 & 0.00 & 416.85 & 0.94 & 416.84 & 0.94 & 412.96 & 0.00 & 412.96 & 0.00 & 416.85 & 0.94 & 415.29 & 0.56 \\
\hline C7 & 11 & 568.49 & 654.27 & 15.09 & 568.49 & 0.00 & $\overline{568.49}$ & 0.00 & 568.49 & 0.00 & 568.49 & 0.00 & $\overline{569.51}$ & 0.18 & $\overline{568.49}$ & 0.00 & 569.51 & 0.18 & 568.69 & 0.04 \\
\hline C8 & 9 & 644.63 & 752.98 & 16.81 & $\overline{647.56}$ & 0.45 & $\overline{645.16}$ & 0.08 & $\overline{645.16}$ & 0.08 & $\overline{645.16}$ & 0.08 & 645.16 & 0.08 & $\overline{645.16}$ & 0.08 & 647.56 & 0.45 & 645.64 & 0.16 \\
\hline C9 & 14 & 756.14 & 896.61 & 18.58 & 756.81 & 0.09 & 756.81 & 0.09 & 757.78 & 0.22 & 756.38 & 0.03 & 759.60 & 0.46 & 756.38 & 0.03 & 759.60 & 0.46 & 757.48 & 0.18 \\
\hline C10 & 17 & 875.07 & 983.97 & 12.44 & 901.18 & 2.98 & 898.16 & 2.64 & 897.99 & 2.62 & 886.75 & 1.33 & 887.69 & 1.44 & 886.75 & 1.33 & 901.18 & 2.98 & 894.35 & 2.20 \\
\hline C11 & 7 & 682.12 & 835.93 & 22.55 & 690.83 & 1.28 & 689.24 & 1.04 & 691.10 & 1.32 & 692.63 & 1.54 & 691.36 & 1.35 & 689.24 & 1.04 & 692.63 & 1.54 & 691.03 & 1.31 \\
\hline C12 & 10 & *534.24 & 545.25 & 2.06 & *534.24 & 0.00 & *534.24 & 0.00 & *534.24 & 0.00 & *534.24 & 0.00 & *534.24 & 0.00 & *534.24 & 0.00 & *534.24 & 0.00 & $* 534.24$ & 0.00 \\
\hline $\mathrm{C} 13$ & 12 & 896.50 & 1025.11 & 14.35 & $9 \overline{902.87}$ & 0.71 & $9 \overline{912.53}$ & 1.79 & $9 \overline{04.17}$ & 0.86 & $9 \overline{05.14}$ & 0.96 & $9 \overline{05.79}$ & 1.04 & $9 \overline{02.87}$ & 0.71 & $9 \overline{12.53}$ & 1.79 & $9 \overline{906.10}$ & 1.07 \\
\hline C14 & 12 & 581.81 & 641.66 & 10.29 & 581.92 & 0.02 & 581.92 & 0.02 & 581.92 & 0.02 & 581.92 & 0.02 & 581.92 & 0.02 & 581.92 & 0.02 & 581.92 & 0.02 & 581.92 & 0.02 \\
\hline F11 & 4 & $* 177.00$ & 201.27 & 13.71 & $* 177.00$ & 0.00 & *177.00 & 0.00 & $* 177.00$ & 0.00 & $* 177.00$ & 0.00 & *177.00 & 0.00 & $* 177.00$ & 0.00 & $* 177.00$ & 0.00 & $* 177.00$ & 0.00 \\
\hline F12 & 7 & 769.66 & 919.22 & 19.43 & $7 \overline{83.41}$ & 1.79 & $7 \overline{84.12}$ & 1.88 & $7 \overline{82.66}$ & 1.69 & $7 \overline{85.35}$ & 2.04 & $7 \overline{84.12}$ & 1.88 & $7 \overline{82.66}$ & 1.69 & $7 \overline{85.35}$ & 2.04 & $7 \overline{83.93}$ & 1.85 \\
\hline Avg. & & & & 19.67 & & 0.73 & & 0.66 & & 0.74 & & 0.67 & & 0.69 & & 0.45 & & 0.98 & & 0.70 \\
\hline $\mathrm{Pb}$ & & & t.time & & t.time & b.time & t.time & b.time & t.time & b.time & t.time & b.time & t.time & b.time & & & & & t.time & b.time \\
\hline $\mathrm{C} 1$ & & & 0.0 & & 70.2 & 20.6 & 72.3 & 14.9 & 65.8 & 33.6 & 61.9 & 14.8 & 61.4 & 25.0 & & & & & 66.3 & 21.8 \\
\hline C2 & & & 0.1 & & 86.2 & 62.2 & 78.2 & 28.7 & 94.3 & 32.0 & 83.0 & 58.5 & 77.2 & 52.1 & & & & & 83.8 & 46.7 \\
\hline C3 & & & 0.1 & & 136.4 & 69.1 & 110.7 & 98.4 & 110.3 & 37.6 & 119.8 & 68.3 & 112.0 & 36.0 & & & & & 117.8 & 61.9 \\
\hline C4 & & & 0.2 & & 177.8 & 127.5 & 159.2 & 88.3 & 188.9 & 148.5 & 186.4 & 64.3 & 177.5 & 79.1 & & & & & 178.0 & 101.5 \\
\hline C5 & & & 0.4 & & 271.1 & 269.7 & 269.1 & 224.8 & 291.6 & 173.5 & 262.1 & 152.7 & 268.2 & 238.2 & & & & & 272.4 & 211.8 \\
\hline C6 & & & 0.0 & & 48.5 & 0.6 & 44.0 & 26.8 & 51.1 & 0.6 & 50.6 & 0.5 & 45.9 & 10.7 & & & & & 48.0 & 7.8 \\
\hline C7 & & & 0.1 & & 85.0 & 68.3 & 75.6 & 15.5 & 81.3 & 13.7 & 75.3 & 43.2 & 75.7 & 23.0 & & & & & 78.6 & 32.7 \\
\hline C8 & & & 0.1 & & 153.1 & 87.3 & 160.8 & 123.6 & 169.6 & 29.2 & 153.3 & 51.3 & 151.7 & 110.5 & & & & & 157.7 & 80.4 \\
\hline C9 & & & 0.2 & & 295.2 & 138.5 & 298.2 & 200.5 & 317.3 & 250.9 & 281.9 & 258.4 & 304.3 & 260.2 & & & & & 299.4 & 221.7 \\
\hline C10 & & & 0.4 & & 705.2 & 665.0 & 721.8 & 524.1 & 729.1 & 719.1 & 828.4 & 678.8 & 584.2 & 556.8 & & & & & 713.7 & 628.8 \\
\hline C11 & & & 0.1 & & 219.8 & 145.9 & 176.5 & 45.1 & 248.5 & 217.7 & 227.1 & 145.8 & 227.3 & 194.7 & & & & & 219.8 & 149.8 \\
\hline C12 & & & 0.1 & & 99.6 & 23.0 & 89.8 & 61.3 & 96.2 & 12.5 & 102.2 & 74.2 & 96.6 & 27.9 & & & & & 96.9 & 39.8 \\
\hline C13 & & & 0.1 & & 1113.8 & 393.4 & 1359.0 & 1270.8 & 1105.3 & 787.1 & 845.0 & 603.9 & 1244.1 & 562.0 & & & & & 1133.4 & 723.4 \\
\hline C14 & & & 0.1 & & 363.1 & 213.6 & 327.1 & 124.8 & 486.7 & 421.3 & 305.6 & 159.2 & 452.1 & 325.2 & & & & & 386.9 & 248.8 \\
\hline F11 & & & 0.1 & & 97.9 & 59.1 & 79.8 & 74.7 & 88.9 & 25.7 & 91.1 & 53.0 & 88.2 & 31.6 & & & & & 89.2 & 48.8 \\
\hline F12 & & & 0.2 & & 190.9 & 36.4 & 176.7 & 80.3 & 152.2 & 43.5 & 178.8 & 140.4 & 181.7 & 43.9 & & & & & 176.1 & 68.9 \\
\hline Avg. & & & 0.1 & & 257.1 & 148.8 & 262.4 & 187.7 & 267.3 & 184.2 & 240.8 & 160.5 & 259.3 & 161.1 & & & & & 257.4 & 168.4 \\
\hline
\end{tabular}

CPU times are expressed in seconds. 
Table 5

Current best known solution costs for the tested OVRP benchmark instances.

\begin{tabular}{|c|c|c|c|c|c|c|c|c|c|}
\hline \multirow[t]{3}{*}{ Inst. } & \multirow[t]{3}{*}{$n$} & \multirow[t]{3}{*}{$D$} & \multicolumn{7}{|c|}{ Best known solution } \\
\hline & & & \multicolumn{4}{|c|}{$F=\infty$} & \multicolumn{3}{|c|}{$F=0$} \\
\hline & & & $m$ & LB & Cost & Best heuristics & $m$ & Cost & Best heuristics \\
\hline $\mathrm{C} 1$ & 50 & & 5 & 416.1 & $* 416.06$ & {$[3,8,12-14,18,20]$} & 6 & 412.96 & [24-26] \\
\hline $\mathrm{C} 2$ & 75 & & 10 & 559.62 & 567.14 & {$[8,12-14,18,20]$} & 11 & 564.06 & [24-26] \\
\hline C3 & 100 & & 8 & 639.7 & * $\underline{639.74}$ & {$[8,12,18]$} & 9 & 639.57 & {$[26]$} \\
\hline $\mathrm{C} 4$ & 150 & & 12 & 730.2 & 733.13 & {$[8,12,18,20]$} & & & \\
\hline C5 & 199 & & 16 & 848.5 & $\overline{879.37}$ & {$[25]$} & 17 & 868.44 & \\
\hline C6 & 50 & 180 & 6 & & 412.96 & {$[3,8,12-14,18,20]$} & & & \\
\hline $\mathrm{C} 7$ & 75 & 144 & 10 & & 583.19 & {$[20]$} & 11 & 568.49 & {$[8,13,14,18]$} \\
\hline $\mathrm{C} 8$ & 100 & 207 & 9 & & 644.63 & {$[3,8,12,18]$} & & & \\
\hline C9 & 150 & 180 & 13 & & $\overline{757.69}$ & & 14 & 756.14 & {$[8]$} \\
\hline $\mathrm{C} 10$ & 199 & 180 & 17 & & 874.71 & & & & \\
\hline C11 & 120 & & 7 & 657.1 & 682.12 & {$[8,12,20]$} & 10 & 678.54 & {$[26]$} \\
\hline C12 & 100 & & 10 & 534.2 & *534.24 & {$[8,12,18,20,24-26]$} & & & \\
\hline C13 & 120 & 648 & 11 & & 899.16 & & 12 & 894.19 & \\
\hline C14 & 100 & 936 & 11 & & 591.87 & {$[8,12,18,20]$} & 12 & 581.81 & {$[8]$} \\
\hline F11 & 71 & & 4 & 177.0 & *177.00 & {$[8,13,14,18,20]$} & & & \\
\hline F12 & 134 & & 7 & 762.9 & $7 \overline{69.55}$ & & & & \\
\hline 01 & 200 & & 5 & & 6018.52 & {$[8,18]$} & & & \\
\hline $\mathrm{O} 2$ & 240 & & 9 & & 4573.53 & & & & \\
\hline $\mathrm{O} 3$ & 280 & & 7 & & 7731.46 & [8] & & & \\
\hline $\mathrm{O} 4$ & 320 & & 10 & & 7251.74 & & & & \\
\hline O5 & 360 & & 8 & & 9197.61 & [18] & 9 & 9156.74 & \\
\hline 06 & 400 & & 9 & & 9803.80 & [18] & & & \\
\hline 07 & 440 & & 10 & & 10344.37 & & & & \\
\hline 08 & 480 & & 10 & & 12420.16 & [8] & & & \\
\hline
\end{tabular}

algorithm is satisfactory: starting from a set of initial solutions with an average percentage deviation (w.r.t. the best known value) of 19.67, HIP finds a set of final solutions with an average percentage deviation of 0.70 in an average overall computing time of $257.4 \mathrm{~s}$.

The current best known solution costs for the tested instances are given in summary in Table 5, where we also report the number of customers $n$ and the route duration limit $D$ associated with the vehicles. Solution costs are given both for the case $F=\infty$ (i.e., when the objective is to minimize the number of used vehicles first and the traveling cost second) and the case $F=0$ (i.e., when the objective is to minimize the traveling cost). As usual, the best known solution cost for the case $F=0$ is reported only if the traveling cost is smaller than the corresponding one for the case $F=\infty$. For each instance whose best known solution was not improved by HIP we report the algorithms providing the corresponding best known costs. Previously best known solution costs reached also by HIP (starting from a worse solution) are underlined, while new best solution costs found by HIP are in bold face. For the capacitated instances, in the case $F=\infty$, we also report the best known lower bound $L B$ taken from $[17,19]$.

\section{Conclusions and future directions}

We addressed the Open Vehicle Routing Problem (OVRP), a variant of the "classical" Vehicle Routing Problem (VRP) in which the vehicles are not required to return to the depot after completing their service. OVRP has recently received an increasing attention in the literature, and several heuristic and metaheuristic algorithms have been proposed for this problem, as well as exact approaches.

We presented a heuristic improvement procedure for OVRP based on Integer Linear Programming (ILP) techniques. Given an initial solution to be possibly improved, the method follows a destruct-andrepair paradigm, where the given solution is randomly destroyed (i.e., customers are removed in a random way) and repaired by solving an ILP model, in the attempt of finding a new improved solution.

Computational results on 24 benchmark instances from the literature showed that the proposed improvement method can be used as a profitable tool for finding good-quality OVRP solutions, and that even extremely good quality solutions found by the most effective metaheuristic techniques proposed for OVRP can be improved. Out of 30 best known solutions which are not provably optimal, in 10 cases the proposed method was able to improve on the best known solution reported in the literature.

Future directions of work could involve more sophisticated criteria for removing customers from the current solution, as well as more sophisticated algorithms for solving the column generation problem related to the ILP model. On the other side, the overall procedure can be considered as a general framework and it could be extended to cover other variants of Vehicle Routing Problems, as, for example, Vehicle Routing Problems with heterogenous vehicles and multi-depot Vehicle Routing Problems.

\section{Acknowledgments}

This work has been partially supported by MIUR (Ministero Istruzione, Università e Ricerca), Italy. We wish to thank Zhuo Fu, Richard Eglese, Leon Li, Krzysztof Fleszar, Ibrahim H. Osman, Khalil S. Hindi, David Pisinger, Stefan Ropke, Ulrich Derigs and Katharina Reuter who provided the initial solutions used in the computational experiments. We also thank the referees for their constructive and useful comments and remarks.

\section{Appendix}

New best known solutions found by HIP (see Table A1). 


$\begin{array}{llr}\text { 1: } & 0 & 105 \\ \text { 2: } & 0 & 112 \\ \text { 3: } & 0 & 53 \\ \text { 4: } & 0 & 28 \\ \text { 5: } & 0 & 58 \\ \text { 6: } & 0 & 166 \\ \text { 7: } & 0 & 27 \\ \text { 8: } & 0 & 111 \\ \text { 9: } & 0 & 154 \\ \text { 10: } & 0 & 180 \\ \text { 11: } & 0 & 146 \\ \text { 12: } & 0 & 13 \\ \text { 13: } & 0 & 156 \\ \text { 14: } & 0 & 167 \\ \text { 15: } & 0 & 89 \\ \text { 16: } & 0 & 94 \\ \text { 17: } & 0 & 176\end{array}$

$\begin{array}{rrr}26 & 149 & 195 \\ 183 & 6 & 96 \\ 40 & 21 & 73 \\ 184 & 76 & 196 \\ 152 & 137 & 2 \\ 83 & 199 & 114 \\ 132 & 69 & 162 \\ 50 & 102 & 157 \\ 138 & 12 & 109 \\ 198 & 110 & 4 \\ 52 & 153 & 106 \\ 117 & 151 & 92 \\ 147 & 60 & 118 \\ 127 & 190 & 31 \\ 18 & 82 & 48 \\ 95 & 97 & 87 \\ 1 & 122 & 51\end{array}$

$\begin{array}{rr}179 & \\ 99 & 104 \\ 171 & \\ 116 & \\ 115 & 178 \\ 8 & 174 \\ 101 & \\ 33 & 185 \\ 177 & 150 \\ 155 & 139 \\ 194 & \\ 37 & \\ 5 & \\ 189 & \\ 123 & \\ 172 & \\ 9 & \end{array}$

$\begin{array}{rr}54 & 130 \\ 104 & 59 \\ 74 & 72 \\ 77 & 3 \\ 178 & 144 \\ 174 & 46 \\ 70 & 30 \\ 185 & 81 \\ 150 & 80 \\ 139 & 187 \\ 7 & 182 \\ 98 & 100 \\ 84 & 173 \\ 10 & 108 \\ 19 & 107 \\ 42 & 142 \\ 103 & 161\end{array}$

$\begin{array}{rrr}165 & 55 & 25 \\ 93 & 85 & 6 \\ 197 & 75 & 133 \\ 158 & 79 & 129 \\ 57 & 15 & 43 \\ 124 & 168 & 47 \\ 160 & 128 & 20 \\ 120 & 164 & 34 \\ 68 & 134 & 163 \\ 39 & 56 & 18 \\ 88 & 148 & 62 \\ 193 & 91 & 191 \\ 113 & 17 & 45 \\ 90 & 32 & 13 \\ 175 & 11 & 126 \\ 14 & 192 & 11 \\ 71 & 135 & 35\end{array}$

Problem C9. $m$ : 1 Solution:

$\begin{array}{llr}\text { 1: } & 0 & 1 \\ \text { 2: } & 0 & 139 \\ \text { 3: } & 0 & \\ \text { 4: } & 0 & 1 \\ \text { 5: } & 0 & \\ \text { 6: } & 0 & \\ \text { 7: } & 0 & \\ \text { 8: } & 0 & \\ \text { 9: } & 0 & \\ \text { 10: } & 0 & \\ \text { 11: } & 0 & \\ \text { 12: } & 0 & \\ \text { 13: } & 0 & \\ \end{array}$

$\begin{array}{rrrr}108 & 37 & 52 & 15 \\ 139 & 18 & 110 & 133 \\ 46 & 102 & 6 & 57 \\ 100 & 2 & 83 & 131 \\ 56 & 144 & 146 & 109 \\ 27 & 81 & 138 & 48 \\ 38 & 62 & 9 & 130 \\ 90 & 71 & 123 & 122 \\ 12 & 47 & 68 & 14 \\ 63 & 17 & 145 & 147 \\ 77 & 32 & 119 & 51 \\ 103 & 5 & 76 & 49 \\ 78 & 11 & 126 & 16\end{array}$

oblem C10. m: 17 cost: 874.7

$\begin{array}{rrr}150 & 80 & 134 \\ 30 & 20 & 188 \\ 33 & 81 & 120 \\ 179 & 110 & 155 \\ 124 & 168 & 47 \\ 93 & 85 & 193 \\ 84 & 173 & 61 \\ 178 & 115 & 145 \\ 122 & 51 & 9 \\ 87 & 144 & 57 \\ 177 & 54 & 130 \\ 151 & 92 & 37 \\ 88 & 148 & 123 \\ 83 & 199 & 114 \\ 158 & 185 & 79\end{array}$

163
128
135
4
36
91
16
41
103
172
165
98
19
8
129

24
160
35
139
143
191
86
22
161
42
55
100
107
125
169

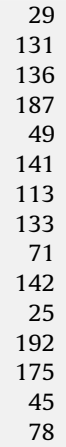
121
32 65 39
64


Table A1. (continued)

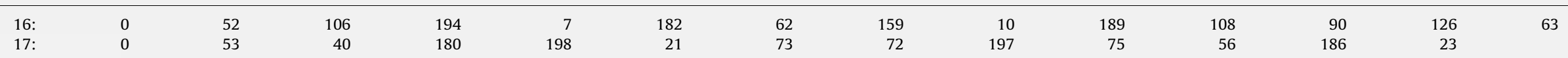

Problem C13. m: 11

cost: 899.16

Solution

$\begin{array}{llr}\text { 1: } & 0 & 87 \\ \text { 2: } & 0 & 112 \\ \text { 3: } & 0 & 98 \\ \text { 4: } & 0 & 119 \\ \text { 5: } & 0 & 67 \\ \text { 6: } & 0 & 110 \\ \text { 7: } & 0 & 21 \\ \text { 8: } & 0 & 95 \\ 9: & 0 & 88 \\ \text { 10: } & 0 & 109 \\ \text { 11: } & 0 & 120\end{array}$

Problem C13. m: 12 cost: 894.19

$\begin{array}{llr}\text { 1: } & 0 & 109 \\ \text { 2: } & 0 & 82 \\ \text { 3: } & 0 & 88 \\ \text { 4: } & 0 & 21 \\ \text { 5: } & 0 & 67 \\ \text { 6: } & 0 & 112 \\ \text { 7: } & 0 & 119 \\ \text { 8: } & 0 & 103 \\ \text { 9: } & 0 & 98 \\ \text { 10: } & 0 & 9 \\ \text { 11: } & 0 & \\ \text { 12: } & 0 & 120\end{array}$

$\begin{array}{rrr}109 & 17 & \\ 82 & 111 & 86 \\ 88 & 87 & 95 \\ 21 & 20 & 23 \\ 67 & 69 & 70 \\ 112 & 84 & \\ 119 & 81 & 117 \\ 103 & 68 & 79 \\ 98 & 52 & 54 \\ 93 & 94 & 97 \\ 96 & 115 & 37 \\ 120 & & \end{array}$

$\begin{array}{rrr}16 & 19 & 25 \\ 86 & 85 & 89 \\ 95 & 102 & 105 \\ 23 & 26 & 28 \\ 70 & 71 & 74 \\ 7 & 9 & 10 \\ 117 & 113 & 83 \\ 79 & 80 & 53 \\ 54 & 57 & 59 \\ 97 & 110 & 40 \\ 37 & 38 & 39\end{array}$

Problem F12. m: 7 cost: 769.55

$\begin{array}{llrrrr}\text { 1: } & 0 & 73 & 74 & 77 & 64 \\ & & 56 & 57 & 105 & 93 \\ \text { 2: } & 0 & 6 & 7 & 8 & 9 \\ \text { 3: } & 0 & 91 & 82 & 19 & 65 \\ \text { 4: } & 0 & 17 & 18 & 25 & 26 \\ \text { 5: } & 0 & 81 & 112 & 126 & 125 \\ \text { 6: } & 0 & 22 & 24 & 23 & 127 \\ \text { 7: } & 0 & 101 & 35 & 36 & 99 \\ & & 46 & 118 & 71 & 66\end{array}$

$\begin{array}{rr}37 & 38 \\ 7 & 9 \\ 79 & 80 \\ 117 & 113 \\ 70 & 71 \\ 54 & 57 \\ 23 & 26 \\ 94 & 97 \\ 111 & 86 \\ 16 & 19 \\ 106 & 107\end{array}$

$\begin{array}{rr}39 & 42 \\ 10 & 11 \\ 53 & 55 \\ 83 & 6 \\ 74 & 72 \\ 59 & 65 \\ 28 & 32 \\ 115 & 40 \\ 85 & 89 \\ 25 & 22 \\ 104 & 103\end{array}$

41
15
58
5
75
61
35
43
91
24
116

44
14
56
4
78
62
34
45
90
27
100

47
13
60
3
77
64
36
48
114
31
99

$\begin{array}{rr}46 & 49 \\ 12 & 8 \\ 63 & \\ 2 & 1 \\ 76 & 73 \\ 66 & \\ 29 & \\ 51 & 50 \\ 18 & 118 \\ 30 & 33 \\ 101 & 102\end{array}$

$\begin{array}{rr}25 & \\ 89 & \\ 105 & \\ 28 & \\ 74 & \\ 10 & \\ 83 & \\ 53 & \\ 59 & \\ 40 & \\ 39 & \end{array}$

$\begin{array}{rr}22 & 24 \\ 92 & 91 \\ 106 & 107 \\ 32 & 35 \\ 75 & 72 \\ 11 & 15 \\ 6 & 5 \\ 55 & 58 \\ 65 & 61 \\ 43 & 45 \\ 42 & 41\end{array}$

27
90
104
34
78
14
4
56
62
48
44

$\begin{array}{rr}31 & 30 \\ 114 & 18 \\ 101 & 99 \\ 36 & 29 \\ 77 & 76 \\ 13 & 12 \\ 3 & 2 \\ 60 & 63 \\ 64 & 66 \\ 51 & 50 \\ 47 & 46\end{array}$

Problem 02. m: 9 cost: 4573.53

\begin{tabular}{|c|c|c|c|c|c|c|c|c|c|c|c|c|c|c|c|c|c|}
\hline \multirow[t]{2}{*}{ 1: } & 0 & 16 & 56 & 55 & 95 & 135 & 134 & 133 & 132 & 131 & 130 & 170 & 171 & 172 & 173 & 174 & 175 \\
\hline & & 176 & 177 & 178 & 179 & 180 & 181 & 182 & 183 & 184 & 185 & & & & & & \\
\hline \multirow[t]{2}{*}{ 2: } & 0 & 42 & 43 & 44 & 84 & 83 & 82 & 122 & 121 & 161 & 162 & 202 & 201 & 240 & 239 & 238 & 237 \\
\hline & & 236 & 235 & 234 & 233 & 232 & 231 & 230 & 229 & 228 & 227 & & & & & & \\
\hline \multirow[t]{2}{*}{ 3: } & 0 & 18 & 17 & 57 & 97 & 96 & 136 & 137 & 138 & 139 & 140 & 141 & 142 & 143 & 144 & 145 & 146 \\
\hline & & 147 & 148 & 149 & 150 & 151 & 152 & 153 & 154 & 155 & 156 & 157 & 158 & 159 & & & \\
\hline 4: & 0 & 13 & 53 & 52 & 51 & 50 & 49 & 48 & 47 & 46 & 45 & 85 & 86 & 87 & 127 & 126 & 125 \\
\hline
\end{tabular}




\begin{tabular}{|c|c|c|c|c|c|c|c|c|c|c|c|c|c|c|c|c|c|c|}
\hline \multirow{10}{*}{ 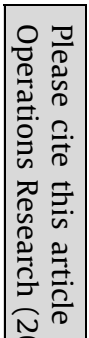 } & \multirow{2}{*}{ 5: } & \multirow{2}{*}{0} & $\begin{array}{r}124 \\
15\end{array}$ & $\begin{array}{r}123 \\
14\end{array}$ & $\begin{array}{r}163 \\
54\end{array}$ & $\begin{array}{r}164 \\
94\end{array}$ & $\begin{array}{r}165 \\
93\end{array}$ & $\begin{array}{r}166 \\
92\end{array}$ & $\begin{array}{r}206 \\
91\end{array}$ & $\begin{array}{r}205 \\
90\end{array}$ & $\begin{array}{r}204 \\
89\end{array}$ & $\begin{array}{r}203 \\
88\end{array}$ & 128 & \multirow{2}{*}{129} & \multirow{2}{*}{169} & \multirow{2}{*}{168} & \multirow{2}{*}{167} & \multirow{2}{*}{207} \\
\hline & & & 208 & 209 & 210 & 211 & 212 & 213 & 214 & 215 & 216 & 217 & 218 & & & & & \\
\hline & 6: & 0 & 25 & 24 & 23 & 22 & 21 & 20 & 60 & 61 & 62 & 63 & 64 & 65 & 66 & 67 & 68 & 69 \\
\hline & & & 70 & 71 & 72 & 73 & 74 & 75 & 76 & 77 & 78 & 79 & 80 & & & & & \\
\hline & 7: & 0 & 1 & 41 & 81 & 120 & 160 & 200 & 199 & 198 & 197 & 196 & 195 & 194 & 193 & 192 & 191 & 190 \\
\hline & & & 189 & 188 & 187 & 186 & 226 & 225 & 224 & 223 & 222 & 221 & 220 & 219 & & & & \\
\hline & 8: & 0 & 19 & 59 & 58 & 98 & 99 & 100 & 101 & 102 & 103 & 104 & 105 & 106 & 107 & 108 & 109 & 110 \\
\hline & & & 111 & 112 & 113 & 114 & 115 & 116 & 117 & 118 & 119 & & & & & & & \\
\hline & 9: & 0 & 26 & 27 & 28 & 29 & 30 & 31 & 32 & 33 & 34 & 35 & 36 & 37 & 38 & 39 & 40 & 2 \\
\hline & & & 3 & 4 & 5 & 6 & 7 & 8 & 9 & 10 & 11 & 12 & & & & & & \\
\hline
\end{tabular}

Problem 04. m: 10

\begin{tabular}{|c|c|c|c|c|c|c|c|c|c|c|c|c|c|c|c|c|}
\hline \multirow{3}{*}{ 1: $\quad 0$} & 39 & 79 & 78 & 77 & 76 & 75 & 74 & 114 & 115 & 116 & 117 & 118 & 119 & 159 & 160 & 121 \\
\hline & 122 & 162 & 161 & 201 & 241 & 281 & 282 & 242 & 202 & 203 & 243 & 283 & 284 & 244 & 204 & 205 \\
\hline & 245 & 285 & & & & & & & & & & & & & & \\
\hline \multirow[t]{3}{*}{0} & 28 & 68 & 69 & 70 & 71 & 72 & 73 & 113 & 112 & 111 & 110 & 109 & 108 & 148 & 149 & 150 \\
\hline & 151 & 152 & 153 & 154 & 155 & 156 & 157 & 158 & 198 & 199 & 200 & 240 & 280 & 320 & 319 & 279 \\
\hline & 239 & 238 & 278 & 318 & & & & & & & & & & & & \\
\hline \multirow[t]{3}{*}{0} & 24 & 64 & 65 & 105 & 104 & 103 & 102 & 142 & 143 & 144 & 145 & 146 & 186 & 226 & 266 & 306 \\
\hline & 307 & 267 & 227 & 228 & 268 & 308 & 309 & 269 & 229 & 230 & 270 & 310 & 311 & 271 & 231 & 232 \\
\hline & 272 & 312 & & & & & & & & & & & & & & \\
\hline \multirow[t]{2}{*}{0} & 15 & 16 & 17 & 18 & 19 & 20 & 60 & 59 & 58 & 57 & 56 & 55 & 54 & 94 & 95 & 96 \\
\hline & 97 & 98 & 99 & 100 & & & & & & & & & & & & \\
\hline \multirow[t]{3}{*}{0} & 14 & 13 & 53 & 93 & 133 & 134 & 135 & 136 & 176 & 175 & 174 & 173 & 213 & 253 & 293 & 294 \\
\hline & 254 & 214 & 215 & 255 & 295 & 296 & 256 & 216 & 217 & 257 & 297 & 298 & 258 & 218 & 219 & 220 \\
\hline & 260 & 300 & 299 & 259 & & & & & & & & & & & & \\
\hline \multirow[t]{3}{*}{0} & 25 & 26 & 27 & 67 & 66 & 106 & 107 & 147 & 187 & 188 & 189 & 190 & 191 & 192 & 193 & 194 \\
\hline & 195 & 196 & 197 & 237 & 277 & 317 & 316 & 276 & 236 & 235 & 275 & 315 & 314 & 274 & 234 & 233 \\
\hline & 273 & 313 & & & & & & & & & & & & & & \\
\hline \multirow[t]{2}{*}{0} & 11 & 51 & 50 & 49 & 48 & 47 & 46 & 45 & 44 & 43 & 42 & 41 & 80 & 120 & 81 & 82 \\
\hline & 83 & 84 & 124 & 123 & 163 & 164 & 165 & 166 & 167 & 207 & 206 & 246 & 286 & 287 & 247 & \\
\hline \multirow[t]{3}{*}{ 8: $\quad 0$} & 21 & 22 & 23 & 63 & 62 & 61 & 101 & 141 & 140 & 139 & 138 & 137 & 177 & 178 & 179 & 180 \\
\hline & 181 & 182 & 183 & 184 & 185 & 225 & 265 & 305 & 304 & 264 & 224 & 223 & 263 & 303 & 302 & 262 \\
\hline & 222 & 221 & 261 & 301 & & & & & & & & & & & & \\
\hline \multirow[t]{2}{*}{ 9: } & 10 & 9 & 8 & 7 & 6 & 5 & 4 & 3 & 2 & 1 & 40 & 38 & 37 & 36 & 35 & 34 \\
\hline & 33 & 32 & 31 & 30 & 29 & & & & & & & & & & & \\
\hline \multirow[t]{3}{*}{ 10: } & 12 & 52 & 92 & 91 & 90 & 89 & 88 & 87 & 86 & 85 & 125 & 126 & 127 & 128 & 129 & 130 \\
\hline & 131 & 132 & 172 & 171 & 170 & 169 & 168 & 208 & 248 & 288 & 289 & 249 & 209 & 210 & 250 & 290 \\
\hline & 291 & 251 & 211 & 212 & 252 & 292 & & & & & & & & & & \\
\hline
\end{tabular}

Problem 05. $m: 9$

Solution:

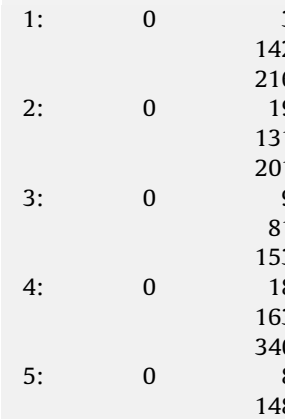

$\begin{array}{rrrrrrrrr}39 & 38 & 37 & 73 & 108 & 107 & 106 & 105 & 104 \\ 143 & 144 & 109 & 145 & 180 & 179 & 215 & 251 & 287 \\ 181 & 217 & 253 & 289 & 325 & & & & \\ 56 & 57 & 58 & 59 & 60 & 96 & 95 & 94 & \\ 132 & 168 & 167 & 166 & 165 & 164 & 200 & 236 & 272 \\ 202 & 238 & 274 & 310 & 346 & 347 & 311 & 275 & 239 \\ 10 & 11 & 12 & 13 & 49 & 48 & 47 & 46 & 45 \\ 82 & 83 & 84 & 85 & 121 & 120 & 119 & 118 & 117 \\ 189 & 225 & 261 & 297 & 333 & 332 & 296 & 260 & 224 \\ 55 & 54 & 53 & 52 & 88 & 89 & 90 & 126 & 125 \\ 199 & 235 & 271 & 307 & 343 & 342 & 306 & 270 & 234 \\ 304 & 268 & 232 & 196 & 195 & 231 & 267 & 303 & 339 \\ 7 & 6 & 5 & 4 & 40 & 41 & 77 & 76 & 751 \\ 147 & 146 & 182 & 218 & 254 & 290 & 326 & 327 & 291\end{array}$

104
287
93
272
239
45
117
224
125
234
339
75
291
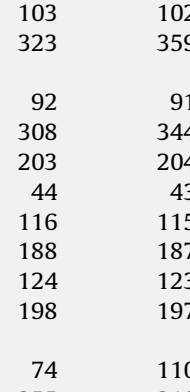

138

127
345

345
240
42
114

114
223
159

159
233

255

$219 \quad 183$

$183 \quad 184$

$\begin{array}{rrr}139 & 140 & 141 \\ 324 & 288 & 252 \\ & & \\ 128 & 129 & 130 \\ 309 & 273 & 237 \\ 276 & 312 & 348 \\ 78 & 79 & 80 \\ 150 & 151 & 152 \\ 259 & 295 & 331 \\ 160 & 161 & 162 \\ 269 & 305 & 341 \\ & & \\ 112 & 113 & 149 \\ 184 & 220 & 256\end{array}$




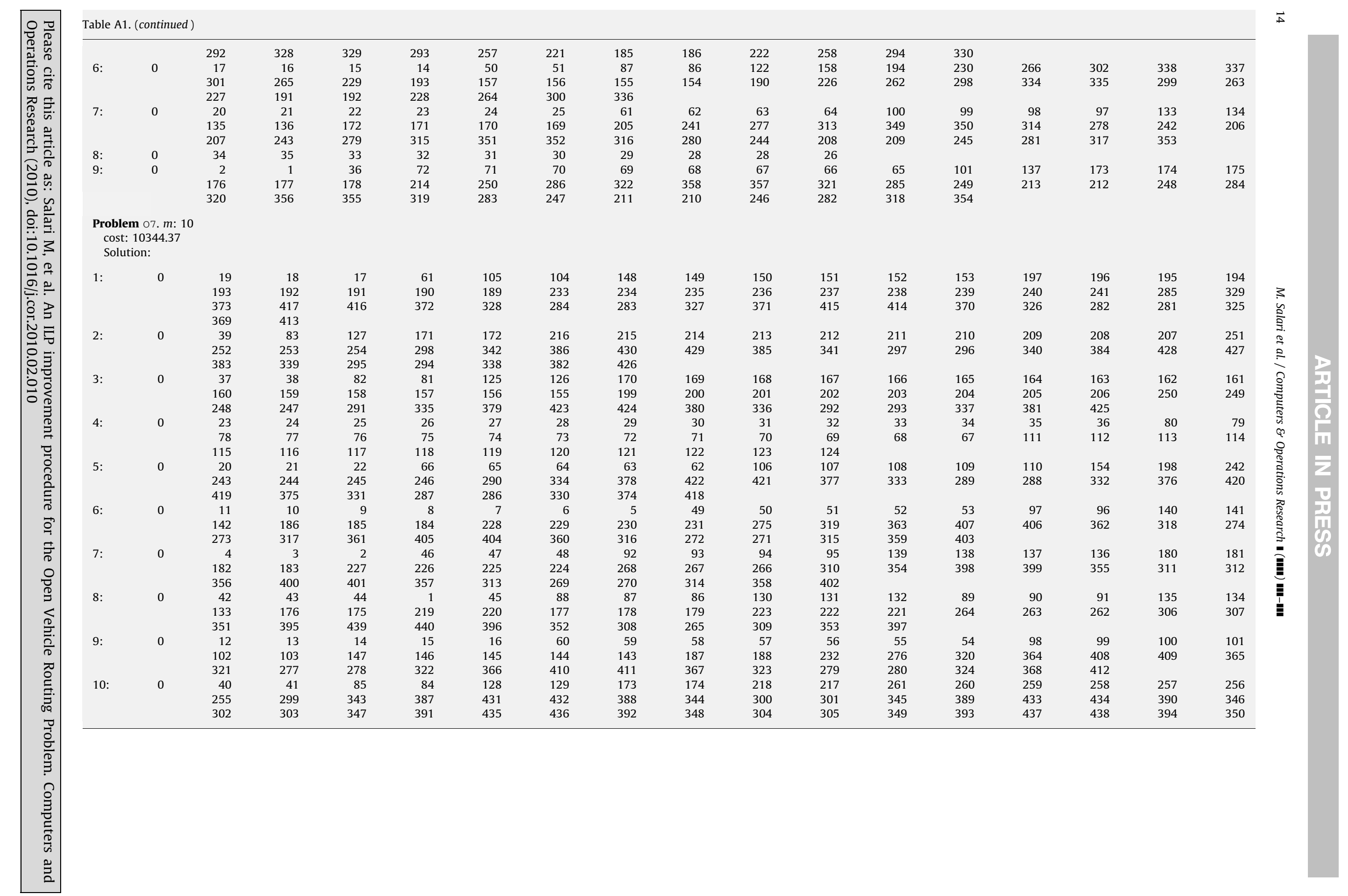




\section{References}

[1] Archetti C, Bertazzi L, Hertz A, Speranza MG. A hybrid heuristic for an inventory-routing problem. Technical Report no. 317, University of Brescia Brescia, Italy, 2009.

[2] Archetti C, Speranza MG, Savelsbergh MWP. An optimization-based heuristic for the split delivery vehicle routing problem. Transportation Science 2008;42:22-31.

[3] Brandão J. A tabu search algorithm for the open vehicle routing problem. European Journal of Operational Research 2004;157:552-64.

[4] Christofides N, Mingozzi A, Toth P. The vehicle routing problem. In: Christofides N, Mingozzi A, Toth P, Sandi C, editors. Combinatorial optimization. Chichester: Wiley; 1979. p. 313-38.

[5] Cordeau J-F, Laporte G, Savelsbergh MWP, Vigo D. Vehicle routing. In: Barnhart C, Laporte G, editors. Transportation. Handbooks in operations research and management science, vol. 14. Amsterdam: Elsevier; 2007. p. 367-428.

[6] Danna E, Rothberg E, Le Pape C. Exploring relaxation induced neighborhoods to improve MIP solutions. Mathematical Programming 2005;102(Ser. A):71-90.

[7] De Franceschi R, Fischetti M, Toth P. A new ILP-based refinement heuristic for vehicle routing problems. Mathematical Programming 2006:105:471-99.

[8] Derigs U, Reuter K. A simple and efficient tabu search heuristic for solving the open vehicle routing problem. Journal of the Operational Research Society 2009; 60:1658-69.

[9] Feillet D, Dejax P, Gendreau M, Gueguen C. An exact algorithm for the elementary shortest path problem with resource constraints: application to some vehicle routing problems. Networks 2004;44:216-29.

[10] Fischetti M, Lodi A. Local branching. Mathematical Programming 2003;98(Ser. B):23-47.

[11] Fisher M. Optimal solutions of vehicle routing problems using minimum k-trees. Operations Research 1994;42:626-42.

[12] Fleszar K, Osman IH, Hindi KS. A variable neighbourhood search for the open vehicle routing problem. European Journal of Operational Research 2009;195:803-9.

[13] Fu Z, Eglese R, Li LYO. A new tabu search heuristic for the open vehicle routing problem. Journal of the Operational Research Society 2005;56:267-74.

[14] Fu Z, Eglese R, Li LYO. Corrigendum: a new tabu search heuristic for the open vehicle routing problem. Journal of the Operational Research Society 2006;57:1018.
[15] Hewitt M, Nemhauser GL, Savelsbergh MWP. Combining exact and heuristic approaches for the capacitated fixed charge network flow problem. INFORMS Journal on Computing 2009, 1-12, doi: 10.1287/ijoc.1090.0348.

[16] IBM ILOG Cplex 〈http://www.ilog.com〉

[17] Letchford AN, Lysgaard J, Eglese RW. A branch-and-cut algorithm for the capacitated open vehicle routing problem. Journal of the Operational Research Society 2007;58:1642-51.

[18] Li F, Golden B, Wasil E. The open vehicle routing problem: algorithms, largescale test problems, and computational results. Computers \& Operations Research 2007;34:2918-30.

[19] Pessoa A, Poggi de Aragão M, Uchoa E. Robust branch-cut-and-price algorithms for vehicle routing problems. In: Golden B, Raghavan S, Wasil E editors. The vehicle routing problem: latest advances and new challenges. New York: Springer; 2008. p. 297-325.

[20] Pisinger D, Ropke S. A general heuristic for vehicle routing problems. Computers \& Operations Research 2007;34:2403-35.

[21] Righini G, Salani M. New dynamic programming algorithms for the resource constrained elementary shortest path problem. Networks 2008 155-70.

[22] Sariklis D, Powell S. A heuristic method for the open vehicle routing problem. Journal of the Operational Research Society 2000;51:564-73.

[23] Schrage L. Formulation and structure of more complex/realistic routing and scheduling problems. Networks 1981;11:229-32.

[24] Tarantilis CD, Diakoulaki D, Kiranoudis CT. Combination of geographical information system and efficient routing algorithms for real life distribution operations. European Journal of the Operational Research 2004;152:437-53.

[25] Tarantilis CD, Ioannou G, Kiranoudis CT, Prastacos GP. A threshold accepting approach to the open vehicle routing problem. RAIRO Operations Research 2004;38:345-60.

[26] Tarantilis CD, Ioannou G, Kiranoudis CT, Prastacos GP. Solving the open vehicle routing problem via a single parameter metaheuristic algorithm. Journal of the Operational Research Society 2005;56:588-96.

[27] Toth P, Tramontani A. An integer linear programming local search for capacitated vehicle routing problems. In: Golden B, Raghavan S, Wasil E editors. The vehicle routing problem: latest advances and new challenges. New York: Springer; 2008. p. 275-95.

[28] Toth P, Vigo D. The vehicle routing problem. SIAM Monographs on Discrete Mathematics and Applications. Philadelphia: SIAM; 2002. 